\title{
MERSIONS OF TOPOLOGICAL MANIFOLDS
}

\author{
BY \\ DAVID GAULD $\left({ }^{1}\right)$
}

\begin{abstract}
We here generalise the immersion and submersion theorems of Smale, Hirsch, Haefliger and Poenaru, Phillips, Lees, and Lashof, giving a relative version in the case of mersions of topological manifolds. A mersion is a map of manifolds $M^{m} \rightarrow Q^{q}$ which in the appropriate local coordinate systems has the form $R^{m} \rightarrow R^{q}$ of the standard inclusion or projection of one euclidean space in another. Such a mersion induces a map of tangent bundles satisfying certain properties. In this paper the problem of classifying mersions is reduced to that of classifying such bundle maps.
\end{abstract}

1. Introduction, definitions and notation. $m, n$ and $q$ will always denote positive integers with $n=\max (m, q)$. The following theorem is proven.

THEOREM 1. Let $M^{m}, M^{\prime n}$ and $Q^{q}$ be manifolds with $M$ a locally flat submanifold of Int $M^{\prime}$. Let $N$ be a closed subset of $M$. Suppose either $m<q$ or that every component of $M-N$ whose closure in $M^{\prime}$ is compact may be connected to $M^{\prime}-M$ by a path lying in $M^{\prime}-N$. Let $\theta: U \rightarrow Q$ be a mersion of a neighbourhood of $N$ in $M^{\prime}$. Then the map

$$
d: \mathscr{M}_{\theta}(M, Q) \rightarrow \mathscr{R}_{\theta}(M, Q)
$$

induced by the differential is a homotopy equivalence.

An immediate corollary is the following classification theorem, cf. Smale [10], Hirsch [4], Haefliger and Poenaru [3], Phillips [8], Lees [6] and Lashof [5].

TheOREM 2. Let $M, M^{\prime}, N, Q$ and $\theta$ be as in Theorem 1. Then the correspondence which assigns to a mersion $f: V \rightarrow Q$ (where $V$ is a neighbourhood of $M$ in $M^{\prime}$ and $f$ agrees with $\theta$ on a neighbourhood of $N$ in $M^{\prime}$ ) its differential df: $T V \rightarrow T Q$ induces a bijection between regular homotopy classes relative to $\theta$ of germs of mersions of $M$ in $Q$ and homotopy classes relative to $\theta$ of representations from $T M$ to $T Q$.

Definition. Let $X$ be a topological space and $M^{m}$ and $Q^{q}$ be topological manifolds. Then a map $f: X \times M \rightarrow X \times Q$ is said to have the uniform mersion property

Received by the editors August 20, 1969.

AMS Subject Classifications. Primary 5570, 5730.

Key Words and Phrases. Classification of immersions, classification of submersions, tangent bundles, differential of an immersion, differential of a submersion, realisation of regular homotopy by isotopy.

(1) This research was partially supported by the NSF under grant GP-6530. 
with respect to $X$ if and only if for every $(x, y) \in X \times M, \exists$ a neighbourhood $U$ of $x$ in $X$ and local charts

$$
h: U \times R^{m} \rightarrow U \times M, \quad g: U \times R^{q} \rightarrow U \times Q,
$$

such that $h$ and $g$ commute with projection on $U$, such that for every $x^{\prime} \in U$, $\left(x^{\prime}, y\right) \in h\left(x^{\prime} \times R^{m}\right)$, and such that the composition

$$
g^{-1} f h: U \times R^{m} \rightarrow U \times R^{q}
$$

is just the map $1 \times \wp$, where $\wp: R^{m} \rightarrow R^{q}$ is projection on the first $q$ coordinates if $m \geqq q$ and is the natural inclusion if $m \leqq q$, i.e. $\wp\left(x_{1}, \ldots, x_{m}\right)=\left(x_{1}, \ldots, x_{m}, 0, \ldots, 0\right)$.

In the case $m \geqq q$, "mersion" means "submersion" and when $m \leqq q$, "mersion" means "immersion". If $X$ is the singleton space, $f$ will be called a mersion (or submersion or immersion).

We now define the two (complete) semisimplicial complexes $\mathscr{M}_{\theta}(M, Q)$ and $\mathscr{R}_{\theta}(M, Q)$ above. A semisimplicial complex is as in May [7], but without degeneracies, cf. Rourke and Sanderson [9]. Let $M^{m}, M^{\prime n}$ and $Q^{q}$ be topological manifolds, with $M$ a locally flat submanifold of Int $M^{\prime}$ and let $N$ be a closed subset of $M$. Suppose given a mersion $\theta: U \rightarrow Q$ of a neighbourhood of $N$ in $M^{\prime}$.

Definition of $\mathscr{M}_{\theta}(M, Q)$. A typical $k$-simplex $F$ of $\mathscr{M}_{\theta}(M, Q)$ is a germ of mersions $f$ as follows:

$$
f: \Delta^{k} \times V \rightarrow \Delta^{k} \times Q,
$$

where $V$ is a neighbourhood of $M$ in $M^{\prime}$, must have the uniform mersion property with respect to the standard $k$-simplex $\Delta^{k}$, and must agree with $1_{\Delta} \times \theta$ on $\Delta^{k} \times$ (some neighbourhood of $N$ in $M^{\prime}$ ). Another such map

$$
f^{\prime}: \Delta^{k} \times V^{\prime} \rightarrow \Delta^{k} \times Q
$$

determines the same $k$-simplex $F$ if and only if $\exists$ a neighbourhood $W$ of $M$ in $M^{\prime}$ such that $f\left|\Delta^{k} \times W=f^{\prime}\right| \Delta^{k} \times W$.

Definition of $\mathscr{R}_{\theta}(M, Q)$. This complex consists of germs of representations from $T M$ to $T Q$. Precisely, a $k$-simplex of $\mathscr{R}_{\theta}(M, Q)$ is represented by a pair $(\Phi, \varphi)$ as in the following commutative diagram:

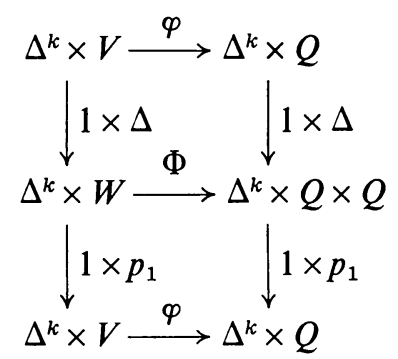

where $V$ is an open neighbourhood of $M$ in $M^{\prime}, \Delta: X \rightarrow X \times X$ is the diagonal map, i.e. $\Delta(x)=(x, x)$, and $W$ is a neighbourhood of $\Delta(V)$ in $V \times V . \varphi$ and $\Phi$ 
must satisfy several additional properties: they must commute with projection on the $\Delta^{k}$ factor, $\Phi$ must agree with $1 \times \theta \times \theta$ on $\Delta^{k} \times($ a neighbourhood of $\Delta(N)$ in $\left.M^{\prime} \times M^{\prime}\right)$, and the map

$$
\Delta^{k} \times W \rightarrow \Delta^{k} \times V \times Q
$$

given by

$$
\left(t, u, u^{\prime}\right) \mapsto\left(t, u, p_{3} \Phi\left(t, u, u^{\prime}\right)\right)
$$

must satisfy the uniform mersion property with respect to $\Delta^{k}$. The last condition essentially says that $\Phi$ is a mersion on each fibre. Another such pair $\left(\Phi^{\prime}, \varphi^{\prime}\right)$ determines the same $k$-simplex if and only if $\Phi^{\prime}$ agrees with $\Phi$ on $\Delta^{k} \times($ a neighbourhood of $\Delta(M)$ in $\left.M^{\prime} \times M^{\prime}\right)$. Note that $(\Phi, \varphi)$ is actually determined by $\Phi$.

Face operations are defined by restriction to a particular face. Each of the above complexes is a Kan complex. If $N=\varnothing$, we will omit the subscript $\theta$.

DEFINITION. The differential

$$
d: \mathscr{M}_{\theta}(M, Q) \rightarrow \mathscr{R}_{\theta}(M, Q)
$$

is defined as follows. Let $f \in F \in \mathscr{M}_{\theta}(M, Q)$, say

$$
f: \Delta^{k} \times V \rightarrow \Delta^{k} \times Q .
$$

Define $\Phi: \Delta^{k} \times V \times V \rightarrow \Delta^{k} \times Q \times Q$ by $\Phi\left(t, u, u^{\prime}\right)=\left(t, p_{2} f(t, u), p_{2} f\left(t, u^{\prime}\right)\right)$. Then define $d(F)$ to be that $k$-simplex of $\mathscr{R}_{\theta}(M, Q)$ determined by $\Phi$.

Definition. Two mersions $f, f^{\prime}: V \rightarrow Q$, where $V$ is a neighbourhood of $M$ in $M^{\prime}$, such that $f$ and $f^{\prime}$ agree with $\theta$ on some neighbourhood of $N$, are regularly homotopic relative to $\theta$ if and only if $\exists$ a mersion

$$
F: I \times V \rightarrow I \times Q
$$

satisfying the uniform mersion property with respect to $I$ such that $F(0, x)=f(x)$, $F(1, x)=f^{\prime}(x)$, and $F\left(t, x^{\prime}\right)=\left(t, \theta\left(x^{\prime}\right)\right)$ for all $x \in V$ and for all $x^{\prime} \in$ some neighbourhood of $N$.

Note that if $f$ and $f^{\prime}$ are regularly homotopic relative to $\theta$ then they determine the vertices of some 1-simplex of $\mathscr{M}_{\theta}(M, Q)$. On the other hand, if $f$ and $f^{\prime}$ determine vertices of the same 1-simplex of $\mathscr{M}_{\theta}(M, Q)$ then the restrictions of $f$ and $f^{\prime}$ to some neighbourhood of $M$ in $M^{\prime}$ are regularly homotopic relative to $\theta$.

DEFINITION. Two representations

$$
(\Phi, \varphi),\left(\Phi^{\prime}, \varphi^{\prime}\right): T M \rightarrow T Q
$$

are homotopic relative to $\theta$ if and only if they determine the vertices of the same 1 -simplex of $\mathscr{R}_{\theta}(M, Q)$.

It is now clear that the two sets referred to in Theorem 2 are merely the pathcomponents of the complexes $\mathscr{M}_{\theta}(M, Q)$ and $\mathscr{R}_{\theta}(M, Q)$.

We now give an idea of the proof. The result is firstly proven in the case where $M$ is obtained from $N$ by adding handles of index $<n$ (see $\$ 3$ for the definition). 
Suppose that $M_{0}$ is a submanifold of $M$ such that $M$ is obtained from $M_{0}$ by adding a handle and $N \subset M_{0}$. In $\S 5$ we prove that the inclusion map $i: M_{0} \rightarrow M$ gives rise to a Kan fibration $i^{*}: \mathscr{R}_{\theta}(M, Q) \rightarrow \mathscr{R}_{\theta}\left(M_{0}, Q\right)$.

In $\S 6$, machinery is set up to enable us, in $\S 7$, to prove that if the handle added to $M_{0}$ to give $M$ is of index $<n$, then the natural restriction

$$
i^{*}: \mathscr{M}_{\theta}(M, Q) \rightarrow \mathscr{M}_{\theta}\left(M_{0}, Q\right)
$$

is a Kan fibration. Using these results together with Theorem 1 in the case $M=B^{m}$, $N=\varnothing$, which is proven in $\S 4$, we deduce, in $\S 3$, that the map $d$ restricts to a homotopy equivalence on each fibre of the fibration induced by $\partial B^{k} \times B^{m-k} \subset B^{k} \times B^{m-k}$, i.e. that $d: \mathscr{M}_{\eta}\left(B^{k} \times B^{m-k}, Q\right) \rightarrow \mathscr{R}_{\eta}\left(B^{k} \times B^{m-k}, Q\right)$ is a homotopy equivalence when $k<n$, where $\eta$ is any mersion of a neighbourhood of $\partial B^{k} \times B^{m-k}$ in $R^{n}$.

Looking at the exact sequences of the above two fibrations and connecting them by $d$, we then have an inductive method for showing that $d: \mathscr{M}_{\theta}(M, Q)$ $\rightarrow \mathscr{R}_{\theta}(M, Q)$ is a weak homotopy equivalence and hence a homotopy equivalence; the induction being on the number of handles making up $M-N$. Details appear in $\S 3$.

$\S 8$ considers the general case. $\mathrm{Cl}(M-N)$ is covered by coordinate patches, each of which has a handle-body structure. The relative theorem is then essentially applied to each of the patches in turn.

The present paper is the major part of my doctoral dissertation written at the University of California at Los Angeles under the direction of Professor Robion C. Kirby. I wish to express deep gratitude to Professor Kirby for his advice and encouragement.

\section{Preliminary results.}

LeMma 3. Let $K$ be a finite simplicial complex. Then a semisimplicial map $\alpha: K \rightarrow \mathscr{M}_{\theta}(M, Q)$ determines a mersion $f: K \times V \rightarrow K \times Q$, where $V$ is a neighbourhood of $M$ in $M^{\prime}$ such that $f$ agrees with $1 \times \theta$ on $K \times\left(\right.$ a neighbourhood of $N$ in $\left.M^{\prime}\right)$ and $f$ satisfies the uniform mersion property with respect to $K$.

A similar result is valid if $\mathscr{M}_{\theta}(M, Q)$ is replaced by $\mathscr{R}_{\theta}(M, Q), f$ now being a representation.

Outline of Proof. For the first case, choose representative mersions $f_{\sigma}$ of the germ $\alpha(\sigma)$ for every simplex $\sigma$ belonging to a sufficiently fine subdivision of $K$, the subdivision being fine enough so that $\alpha(\sigma)$ is a simplex of $\mathscr{M}_{\theta}(M, Q)$. Since $\alpha$ is semisimplicial, one can choose a small enough neighbourhood $V$ of $M$ in $M^{\prime}$ (using finiteness of $K)$ so that $f_{\sigma}$ and $f_{\tau}$ agree on $(\sigma \cap \tau) \times V . f$ is then defined by gluing the $f_{\sigma}$ 's together.

Lemma 4. Suppose that

$$
f: X \times M^{m} \rightarrow X \times Q^{q}
$$

satisfies the uniform mersion property with respect to $X$. Then for every $(x, y) \in X \times M$, 
the charts $h$ and $g$ in the definition of the above property can be chosen to satisfy either of the following:

(i) for every $\xi \in U, h(\xi, 0)=(\xi, y)$;

(ii) for every $(\xi, w) \in U \times R^{q}, p_{2} g(\xi, w)=p_{2} g(x, w)$ if $m \geqq q$.

Proof. Let $(x, y) \in X \times M$ be given.

(i) Let $h^{\prime}: U \times R^{m} \rightarrow U \times M$ and $g^{\prime}: U \times R^{q} \rightarrow U \times Q$ be charts furnished by the definition. Define the required charts by

$$
h(\xi, z)=h^{\prime}\left(\xi, z+p_{2} h^{-1}(\xi, y)\right), \quad g(\xi, w)=g^{\prime}\left(\xi, w+\wp p_{2} h^{-1}(\xi, y)\right),
$$

where $\wp: R^{m} \rightarrow R^{q}$ is as before.

(ii) Let $h^{\prime}$ and $g^{\prime}$ be the charts furnished by (i), with $U^{\prime}$ being the corresponding neighbourhood of $x$ in $X$. Let $h_{\xi}^{\prime}: R^{m} \rightarrow M$ be the embedding given by $h_{\xi}^{\prime}(z)$ $=p_{2} h^{\prime}(\xi, z)$. Similarly define a mersion $f_{\xi}$ and an embedding $g_{\xi}^{\prime}$. We must choose $U, h$ and $g$ such that for all $\xi \in U, g_{\xi}=g_{x}$.

Replace $U^{\prime}$ by a smaller neighbourhood $U$ so that if $\xi \in U$ then $g_{x}^{\prime}\left(B^{q}\right) \subset g_{\xi}^{\prime}\left(R^{q}\right)$ and $g_{\xi}^{\prime}(0) \in g_{x}^{\prime}\left(\right.$ Int $\left.B^{q}\right)$. Now define $h_{\xi}: B^{m} \rightarrow M$ to be the composition

$$
B^{m}=B^{q} \times B^{m-q} \stackrel{\left(g_{\xi}^{\prime-1} g_{x}^{\prime}\right) \times 1}{\longrightarrow} R^{q} \times B^{m-q} \subset R^{m} \stackrel{h_{\xi}^{\prime}}{\longrightarrow} M .
$$

Define $g_{\xi}=g_{x}^{\prime}: R^{q} \rightarrow Q$. If we define $g$ and $h$ in the obvious way then they satisfy (ii).

For the purpose of the following lemma, by a "basic" cube in $I^{r} \times I$ we will mean a cube of the form $I_{1} \times \cdots \times I_{r+1}$, where each $I_{i}$ is a closed subinterval of $I=[0,1]$.

LEMMA 5. Suppose that $\left\{I_{1}, \ldots, I_{k}\right\}$ is a collection of basic cubes covering $I^{r} \times I$. Then $\exists$ another collection $\left\{J_{1}, \ldots, J_{l}\right\}$ of basic cubes such that $\left\{J_{j}\right\}$ covers $I^{r} \times I$, such that if we set $K_{j}=\bigcup_{i<j} J_{i} \cup\left(I^{r} \times 0\right)$ then for all $j, J_{j} \cap K_{j}$ is a flat $r$-ball in $\partial J_{j}$, and that for all $j \in\{1, \ldots, l\}, \exists i \in\{1, \ldots, k\}$ so that $J_{j} \subset I_{i}$.

Proof. The proof is suggested by Figure 1 .
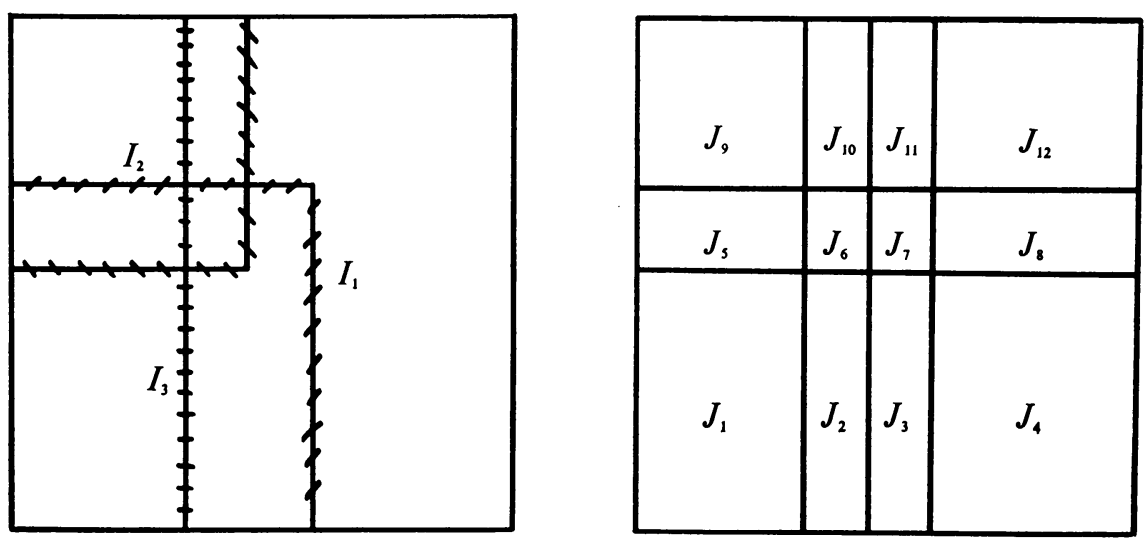

FIGURE 1 
The following result is a consequence of Theorem 12-5 in May [7], the five-lemma, and the exactness of the homotopy sequence of a fibration (Theorem 7.6 in May [7]).

LEMMA 6. Let $p_{i}: E_{i} \rightarrow B_{i}(i=1,2)$ be two fibrations and $f: E_{1} \rightarrow E_{2}$ be a fibre map, i.e. $p_{2} f=f_{0} p_{1}$ for some map $f_{0}: B_{1} \rightarrow B_{2}$. Then any two of the following conditions implies the third:

(i) $f_{0}$ restricts to a homotopy equivalence between the image of $p_{1}$ and that of $p_{2}$;

(ii) $f$ is a homotopy equivalence;

(iii) the restriction of $f$ to each fibre is a homotopy equivalence when considered as a map from the fibre of $E_{1}$ to the corresponding fibre of $E_{2}$.

3. Proof of Theorem 1 when $M-N$ is a handlebody.

Definition. A manifold $M^{m}$ is said to be obtained from the manifold $N^{m}$ by adding $a$ handle of index $k$ or a $k$-handle if and only if $\exists$ an embedding

$$
h: \partial B^{k} \times B^{m-k} \rightarrow \partial N
$$

such that $M$ is obtained from the disjoint union of $N$ and $B^{k} \times B^{m-k}$ by identifying $(x, y) \in \partial B^{k} \times B^{m-k}$ with $h(x, y) \in \partial N$.

We will say that $M-N$ is a handlebody if $\exists$ a sequence $N=M_{0}, M_{1}, \ldots, M_{l}=M$, with $l \leqq \infty$, such that $M_{i}$ is obtained from $M_{i-1}$ by adding a handle.

The proofs of Lemmas 7-9 below appear in later sections.

Lemma 7. The map $d: \mathscr{M}\left(B^{m}, Q\right) \rightarrow \mathscr{R}\left(B^{m}, Q\right)$ is a homotopy equivalence.

Now suppose that $M_{0}^{m}$ is a submanifold of $M$ such that $M$ is obtained from $M_{0}$ by adding finitely many handles and such that $N \subset M_{0}$. Then the inclusion $i: M_{0} \hookrightarrow M$ induces natural restriction maps

$$
i^{*}: \mathscr{R}_{\theta}(M, Q) \rightarrow \mathscr{R}_{\theta}\left(M_{0}, Q\right) \text { and } i^{*}: \mathscr{M}_{\theta}(M, Q) \rightarrow \mathscr{M}_{\theta}\left(M_{0}, Q\right) \text {. }
$$

LeMmA 8. $i^{*}: \mathscr{R}_{\theta}(M, Q) \rightarrow \mathscr{R}_{\theta}\left(M_{0}, Q\right)$ is a fibration.

Lemma 9. $i^{*}: \mathscr{M}_{\theta}(M, Q) \rightarrow \mathscr{M}_{\theta}\left(M_{0}, Q\right)$ is a fibration if $M-M_{0}$ has no n-handles.

LEMMA 10. $d: \mathscr{M}_{\eta}\left(B^{k} \times B^{m-k}, Q\right) \rightarrow \mathscr{R}_{\eta}\left(B^{k} \times B^{m-k}, Q\right)$ is a homotopy equivalence if $k<n$, where $\eta$ is a mersion of a neighbourhood of $\partial B^{k} \times B^{m-k}$.

Proof. (Cf. the lemma on p. 81 of [3].) We prove that

$$
d: \mathscr{M}\left(\partial B^{k} \times B^{m-k+1}, Q\right) \rightarrow \mathscr{R}\left(\partial B^{k} \times B^{m-k+1}, Q\right)
$$

is a homotopy equivalence by induction on $k$, Lemma 10 being a consequence of this proof. To start the induction at $k=1$, consider the following commutative square:

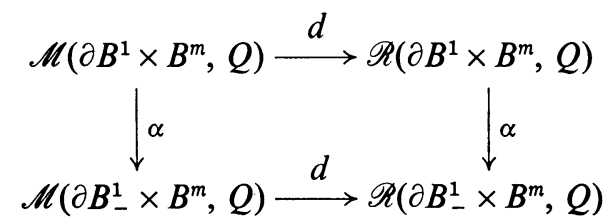


where $\partial B_{-}^{k}$ denotes the collection of points of $\partial B^{k}$ whose last coordinate is nonpositive. $\alpha$ is the restriction map in each case. Note that $\partial B_{-}^{1} \times B^{m}$ is a subhandlebody of $\partial B^{1} \times B^{m}$. Hence by Lemmas 8 and 9 , each of the maps $\alpha$ is a fibration. Now apply Lemma 6. Condition (i) of Lemma 6 is satisfied by Lemma 7 since $\partial B_{-}^{1}$ $\times B^{m} \approx B^{m}$. Condition (iii) is satisfied, since the fibre of $\alpha$ is either $\mathscr{M}_{\eta}\left(B^{0} \times B^{m}, Q\right)$ or $\mathscr{R}_{n}\left(B^{0} \times B^{m}, Q\right)$, i.e. either $\mathscr{M}\left(B^{m}, Q\right)$ or $\mathscr{R}\left(B^{m}, Q\right)$, since $\partial B^{0} \times B^{m}=\varnothing$. Condition (iii) then follows from Lemma 7 . Thus

$$
d: \mathscr{M}\left(\partial B^{1} \times B^{m}, Q\right) \rightarrow \mathscr{R}\left(\partial B^{1} \times B^{m}, Q\right)
$$

is a homotopy equivalence.

Now inductively assume the result for $k \geqq 1, k<n$. Thinking of $\partial B^{k}$ as the equator of $\partial B^{k+1}$, i.e. the boundary of $\partial B_{-}^{k+1}$, we can find a natural inclusion $\partial B^{k} \times B^{1}$ $\subset \partial B_{-}^{k+1}$ as a neighbourhood of $\partial B^{k}$. This gives rise to an inclusion $\partial B^{k} \times B^{m-k+1}$ $\subset \partial B_{-}^{k+1} \times B^{m-k}$. Consider the diagram

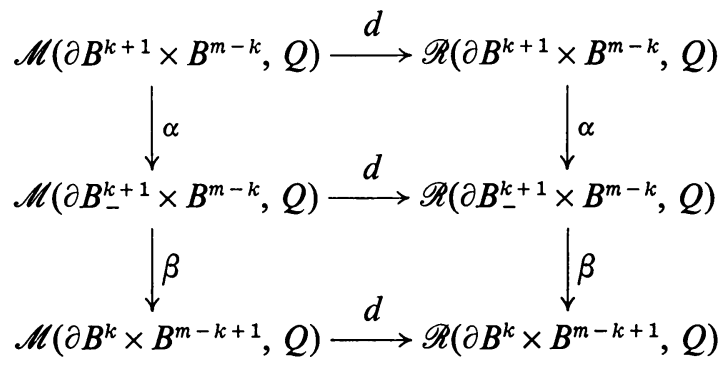

where $\alpha$ is the restriction as above and $\beta$ is the restriction induced by the inclusion. Now $\partial B_{-}^{k+1} \times B^{m-k}$ is obtained from $\partial B^{k} \times B^{m-k+1}$ by adding a handle of index $k$, so by Lemmas 8 and $9, \beta$ is a fibration. By the induction hypothesis and by Lemma 7 respectively, the maps

and

$$
d: \mathscr{M}\left(\partial B^{k} \times B^{m-k+1}, Q\right) \rightarrow \mathscr{R}\left(\partial B^{k} \times B^{m-k+1}, Q\right)
$$

$$
d: \mathscr{M}\left(\partial B_{-}^{k+1} \times B^{m-k}, Q\right) \rightarrow \mathscr{R}\left(\partial B_{-}^{k+1} \times B^{m-k}, Q\right)
$$

are homotopy equivalences. Hence by Lemma 6 the map $d$ is a homotopy equivalence on each fibre. But the fibres are isomorphic to $\mathscr{M}_{n}\left(B^{k} \times B^{m-k}, Q\right)$ and $\mathscr{R}_{\eta}\left(B^{k} \times B^{m-k}, Q\right)$ so that

$$
d: \mathscr{M}_{\eta}\left(B^{k} \times B^{m-k}, Q\right) \rightarrow \mathscr{R}_{\eta}\left(B^{k} \times B^{m-k}, Q\right)
$$

is a homotopy equivalence.

To complete the induction, apply Lemma 6 to the fibrations $\alpha$, noting that condition (i) is satisfied by Lemma 7 and condition (iii) by the reasoning above, since the fibres are isomorphic to $\mathscr{M}_{\eta}\left(B^{k} \times B^{m-k}, Q\right)$ and $\mathscr{R}_{\eta}\left(B^{k} \times B^{m-k}, Q\right)$.

Proof of Theorem 1 in the case where $M$ is obtained from $N$ by adding handles. Suppose inductively that

$$
d: \mathscr{M}_{\theta}\left(M_{0}, Q\right) \rightarrow \mathscr{R}_{\theta}\left(M_{0}, Q\right)
$$


is a homotopy equivalence and that $M$ is obtained from $M_{0}$ by adding a $k$-handle. The hypotheses of the theorem allow us to assume $k<n$, this fact being obvious if $m<q$, and in the case $m \geqq q$, we can find a handlebody decomposition of $M-N$ containing no $n$-handles. Hence by Lemmas 8 and 9 the restrictions $\mathscr{M}_{\theta}(M, Q)$ $\rightarrow \mathscr{M}_{\theta}\left(M_{0}, Q\right)$ and $\mathscr{R}_{\theta}(M, Q) \rightarrow \mathscr{R}_{\theta}\left(M_{0}, Q\right)$ are fibrations. The fibres are isomorphic to $\mathscr{M}_{n}\left(B^{k} \times B^{m-k}, Q\right)$ and $\mathscr{R}_{n}\left(B^{k} \times B^{m-k}, Q\right)$. Thus condition (iii) of Lemma 6 is satisfied by Lemma 10. Condition (i) is satisfied by hypothesis. Hence condition (ii) of Lemma 6 is satisfied, i.e.

$$
d: \mathscr{M}_{\theta}(M, Q) \rightarrow \mathscr{R}_{\theta}(M, Q)
$$

is a homotopy equivalence. This proves the theorem when $M-N$ is a finite handlebody. In the case where $M-N$ requires a countable infinity of handles, the above gives a projective system of homotopy equivalences. On taking the limit, we obtain the required result, cf. Phillips [8, p. 203].

4. Proof of Theorem 1 when $M=B^{m}$. We may assume $M^{\prime}=R^{n}$ with $B^{m}$ embedded in the usual way. Let 0 denote the origin of $R^{n}$. Define $\mathscr{M}(0, Q)$ as before but with $M^{\prime}=R^{n}$ rather than $R^{q}$. Let $\mathscr{R}\left(B_{0}^{n}, Q\right)$ denote the germs of representations taking the fibre over 0 in $T B^{n}$ to a fibre in $T Q$. Then there are natural restriction maps $p: \mathscr{M}\left(B^{m}, Q\right) \rightarrow \mathscr{M}(0, Q)$ and $\rho: \mathscr{R}\left(B^{m}, Q\right) \rightarrow \mathscr{R}\left(B_{0}^{n}, Q\right)$. We thus have a commutative square

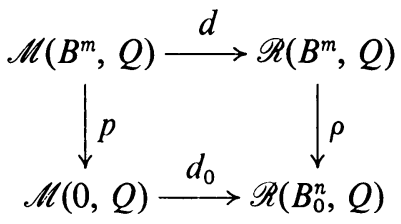

where $d_{0}$ is induced by $d$. The following two lemmas show that $p$ and $\rho$ are homotopy equivalences. It is obvious that $d_{0}$ is an isomorphism, so $d$ must be a homotopy equivalence.

LEMMA 11. $p: \mathscr{M}\left(B^{m}, Q\right) \rightarrow \mathscr{M}(0, Q)$ is a homotopy equivalence.

Proof. We will show that for all $r$, the homomorphism $p_{*}: \pi_{r}\left(\mathscr{M}\left(B^{m}, Q\right)\right)$ $\rightarrow \pi_{r}(\mathscr{M}(0, Q))$ is an isomorphism, the result then following from May [7, Theorem 12-5].

$p_{*}$ is surjective. Suppose given $\alpha: S^{r} \rightarrow \mathscr{M}(0, Q)$, where $\alpha$ is a semisimplicial map. Let

$$
f: S^{r} \times U \rightarrow S^{r} \times Q
$$

be a mersion given by Lemma 3. We may assume that $U$ is an open ball centred at 0 . Let $V$ be a concentric ball of half the radius. Then $\exists$ a natural homeomorphism $r: R^{n} \rightarrow U$ with $r \mid V=1$. Now $f(1 \times r): S^{r} \times R^{n} \rightarrow S^{r} \times Q$ determines a semisimplicial map $\beta: S^{r} \rightarrow \mathscr{M}\left(B^{m}, Q\right)$. Since $r \mid V=1, p \beta=\alpha$. Thus $p_{*}$ is surjective.

$p_{*}$ is injective. Suppose given a semisimplicial map $\alpha: S^{r} \rightarrow \mathscr{M}\left(B^{m}, Q\right)$ such 
that $p \alpha$ is homotopic to a constant map. We must show that $\alpha$ is homotopic to a constant map. The construction of such a homotopy is similar to the construction in the proof that $p_{*}$ is surjective, although it involves two steps rather than one.

LEMMA 12. $\rho: \mathscr{R}\left(B^{m}, Q\right) \rightarrow \mathscr{R}\left(B_{0}^{n}, Q\right)$ is a homotopy equivalence.

Proof. Define a homotopy inverse $\sigma$ as follows: given $f \in F \in \mathscr{R}\left(B_{0}^{n}, Q\right)$, then

$$
f: \Delta^{k} \times 0 \times U \rightarrow \Delta^{k} \times Q \times Q,
$$

where $U$ is a neighbourhood of 0 in $B^{n}$ and $f$ is fibre-preserving, commutes with projection onto $\Delta^{k}$ and is a mersion. Define the neighbourhood $V$ of $\Delta\left(B^{m}\right)$ in $R^{n} \times R^{n}$ by $V=\bigcup_{x \in 2 B^{n}}\{x\} \times(x+U)$, where $x+U=\left\{y \in R^{n} / y-x \in U\right\}$. Define $\Phi: \Delta^{k} \times V \rightarrow \Delta^{k} \times Q \times Q$ by $\Phi(t, x, y)=f(t, 0, y-x)$. Now set $\sigma(F)=[\Phi]$, the germ of $\Phi$. Then $\rho \sigma=1$ and using contractibility of $B^{m}$, one readily shows that $\sigma \rho \simeq 1$.

This completes the proof of Lemma 7.

5. Proof of Lemma 8. We have the following lifting problem:

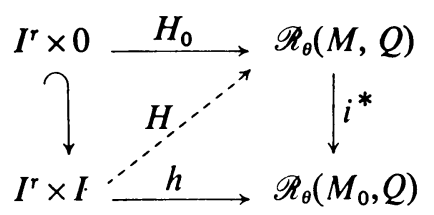

Now by Lemma $3, h$ and $H_{0}$ correspond respectively to representations

$$
\varphi: I^{r} \times I \times T U \rightarrow T Q \text { and } \Phi_{0}: I^{r} \times 0 \times T V \rightarrow T Q,
$$

where $U$ and $V$ are neighbourhoods in $M^{\prime}$ of $M_{0}$ and $M$. We may assume $U \subset V$, and commutativity allows us to assume that $\varphi$ and $\Phi_{0}$ agree on $I^{r} \times 0 \times T U$. Then we can combine $\varphi$ and $\Phi_{0}$ to get a representation

$$
\Phi^{\prime}:\left(I^{r} \times I \times T U\right) \cup\left(I^{r} \times 0 \times T V\right) \rightarrow T Q .
$$

$\left(I^{r} \times I \times T U\right) \cup\left(I^{r} \times 0 \times T V\right)$ is a bundle over $\left(I^{r} \times I \times U\right) \cup\left(I^{r} \times 0 \times V\right)$. We wish to extend $\Phi^{\prime}$ to a representation $\Phi: I^{r} \times I \times T V \rightarrow T Q$. Now the inclusion map

$$
\left(I^{r} \times I \times U\right) \cup\left(I^{r} \times 0 \times V\right) \hookrightarrow I^{r} \times I \times V
$$

is a homotopy equivalence. Let $\rho$ be a homotopy inverse. Then the pull-back under $\rho$ of $\left(I^{r} \times I \times T U\right) \cup\left(I^{r} \times 0 \times T V\right)$ is $I^{r} \times I \times T V$. Let $\hat{\rho}$ be the representation covering $\rho$. Then set $\Phi=\Phi^{\prime} \hat{\rho}$.

6. The isotopy theorem. In this section, we prove the following result.

THEOREM 13. Suppose that $f: \tilde{I}^{r} \times N \rightarrow \tilde{I}^{r} \times Q$ satisfies the uniform mersion property with respect to $\tilde{I}^{r}=[-1,1]^{r}$, where $N^{n}$ and $Q^{q}$ are manifolds with $n \geqq q$. Suppose $M^{n}$ is a compact submanifold of Int $N$. Then $\exists \varepsilon>0$ and a map

$$
H: \varepsilon \tilde{I}^{r} \times N \rightarrow \varepsilon \tilde{I}^{r} \times N
$$


such that:

(i) $H$ is a homeomorphism commuting with projection on $\varepsilon \tilde{I}^{r}$;

(ii) $H \mid \varepsilon \tilde{I}^{r} \times \partial N=1$;

(iii) for all $(t, x) \in \varepsilon \tilde{I}^{r} \times M, p_{2} f(t, x)=p_{2} f\left(0, p_{2} H(t, x)\right)$.

If we define a mersion $f_{t}: N \rightarrow Q$ by $f_{t}(x)=p_{2} f(t, x)$ and a homeomorphism $H_{t}: N \rightarrow N$ by $H_{t}(x)=p_{2} H(t, x)$, then the above result says that for small enough $\varepsilon$ the family of mersions $\left\{f_{t} \mid M / t \in \varepsilon \tilde{I}^{r}\right\}$ can be realised by an isotopy $\left\{H_{t}\right\}$ of $N$ followed by $f_{0}$, i.e. $f_{t}\left|M=f_{0} H_{t}\right| M$. The value of this result is that in the next section we will be able to replace consideration of a regular homotopy of mersions $\left\{f_{t}\right\}$ by consideration of an isotopy $\left\{H_{t}\right\}$ of $N$.

The above result corresponds to Lemma 3.1 in Phillips [8]. Since Phillips uses the result that $\operatorname{Emb}(M, N)$ is an open subset of $\operatorname{Hom}(M, N)$, his proof does not carry over to the PL or topological cases. The proof below uses a topological result of Edwards and Kirby [2] concerning deformations of spaces of embeddings. If the corresponding result in the PL case were true, then, just as in Edwards and Kirby [2] one could deduce that $\mathrm{PL}_{n}$ were locally contractible. The author has shown that local contractibility of both $\mathrm{TOP}_{n}$ and $\mathrm{PL}_{n}$ results in $\mathrm{TOP}_{n}$ and $\mathrm{PL}_{n}$ being homotopy equivalent which is false, see Kirby and Siebenmann [11, p. 748]. Thus the PL result corresponding to the topological results of Edwards and Kirby is false, so that the proof below is invalid in the PL case.

The proof requires several preliminary results.

Definition. Let $C$ and $V$ be subsets of $R^{n}$ with $C$ compact, $V$ open and $C \subset V$. Let $\wp$ be as before. Define $\mathscr{E}_{\wp}\left(V ; R^{n}\right)=\left\{f: V \rightarrow R^{n} / f\right.$ is an embedding and $\left.\wp=\wp f\right\}$.

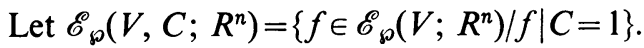

LEMMA 14. With $V$ as above, let $A$ be a compact submanifold of $R^{q}, U$ an open neighbourhood of $A$ in $R^{q}$ such that $U=A \cup_{\partial}[\partial A \times[0,1)], B$ a compact subset of $R^{n-q}$, and $W$ an open set in $R^{n-q}$ containing $B$. Suppose that $(\mathrm{Cl} U) \times(\mathrm{Cl} W) \subset V$. Then given any neighbourhood $\mathscr{Q}$ of the inclusion $\eta: V \hookrightarrow R^{n}$ in $\mathscr{E}_{\mathfrak{p}}\left(V ; R^{n}\right), \exists a$ neighbourhood $\mathscr{P}$ of $\eta$ and a deformation $\varphi: \mathscr{P} \times I \rightarrow \mathscr{Q}$ with $\varphi_{0}=1, \varphi(\mathscr{P} \times 1)$ $\subset \mathscr{E}_{\mathfrak{p}}\left(V, A \times B ; R^{n}\right)$ and $\varphi(f, t)|V-(U \times W)=f| V-(U \times W)$.

Proof. Since neighbourhoods of the form $\mathscr{N}(K, \varepsilon)=\left\{f \in \mathscr{E}_{\mathfrak{p}}\left(V ; R^{n}\right) /\right.$ for all $x \in K$, $|f(x)-x|<\varepsilon\}$ are basic where $K$ runs through compact subsets of $V$ and $\varepsilon$ through positive numbers, we may assume that $\mathscr{Q}=\mathscr{N}(K, \varepsilon)$. Since $A$ and $B$ are compact, we may assume that $(\mathrm{Cl} U) \times(\mathrm{Cl} W)$ is compact. We may assume $\mathrm{Cl} U=A$ $\cup[\partial A \times[0,1]]$. Define the map

$$
\Omega:(\mathrm{Cl} U) \times \mathscr{E}_{\wp}\left(V ; R^{n}\right) \rightarrow \mathscr{E}\left(W ; R^{n-q}\right)
$$

(where $\mathscr{E}\left(W ; R^{n-q}\right)$ is the space of embeddings) by $\Omega(x, f)(y)=\wp^{\prime} f(x, y)$ for all $y \in W$, where $\wp^{\prime}: R^{n} \rightarrow R^{n-q}$ is projection onto the last $n-q$ factors. Let $\mathscr{Q}^{\prime}$ be the $\varepsilon$-neighbourhood of the inclusion map in $\mathscr{E}\left(W ; R^{n-q}\right)$. By Edwards and Kirby [2] 
we can find a neighbourhood $\mathscr{P}^{\prime}$ of the inclusion in $\mathscr{E}\left(W ; R^{n-q}\right)$ and a deformation $\varphi^{\prime}: \mathscr{P}^{\prime} \times I \rightarrow \mathscr{Q}^{\prime}$ such that $\varphi^{\prime}(g, 0)=g, \varphi^{\prime}(g, t)$ agrees with $g$ near $\partial W$, and $\varphi^{\prime}(g, 1) \in \mathscr{E}\left(W, B ; R^{n-q}\right)$. We may suppose that $\mathscr{P}^{\prime}$ is a $\delta$-neighbourhood of the inclusion. Let $\mathscr{P}$ be the neighbourhood $\mathscr{N}(K, \delta)$ of $\eta$ in $\mathscr{E}_{\mathfrak{p}}\left(V ; R^{n}\right)$. Define the deformation $\varphi: \mathscr{P} \times I \rightarrow \mathscr{Q}$ as follows. Let $(f, t) \in \mathscr{P} \times I$ and $(x, y) \in V$, where we think of $V$ as a subset of $R^{q} \times R^{n-q}$. Set

$$
\begin{array}{rlrl}
\varphi(f, t)(x, y) & =f(x, y) & & \text { if }(x, y) \notin U \times W, \\
& =\left(x, \varphi^{\prime}(\Omega(x, f), t)(y)\right) & & \text { if }(x, y) \in A \times W, \\
& =\left(x, \varphi^{\prime}(\Omega(x, f),(1-s) t)(y)\right) & & \text { if } x=\left(x^{\prime}, s\right) \in \partial A \times[0,1], \\
& & \text { and } y \in W .
\end{array}
$$

Then $\mathscr{P}$ and $\varphi$ satisfy the conditions of Lemma 14 .

LEMMA 15. Lemma 14 remains true if we replace $\mathscr{E}_{\wp}\left(V ; R^{n}\right)$ by $\mathscr{E}_{\wp}\left(V, D ; R^{n}\right)$ and $\mathscr{E}_{\wp}\left(V, A \times B ; R^{n}\right)$ by $\mathscr{E}_{\wp}\left(V,(A \times B) \cup D ; R^{n}\right)$ where $D^{n}$ is a compact submanifold of $R^{n}$ and $(A \times B) \cap D=A \times B^{\prime}$ for some $B^{\prime} \subset R^{n-q}$.

Proof. The proof of Lemma 14 carries over if one uses the relative theorem of Edwards and Kirby to get $\varphi^{\prime}$.

LEMMA 16. Given any neighbourhood $\mathscr{Q}$ of the inclusion $\eta: V \hookrightarrow R^{n}, \exists$ a neighbourhood $\mathscr{P}$ of $\eta$ in $\mathscr{E}_{\wp}\left(V ; R^{n}\right)$ and a deformation $\varphi: \mathscr{P} \times I \rightarrow \mathscr{Q}$ of $\mathscr{P}$ into $\mathscr{E}_{\mathfrak{p}}\left(V, C ; R^{n}\right)$; $V$ and $C$ as above.

Proof. Thinking of $R^{n}$ as the product $R^{q} \times R^{n-q}$, we have, for all $x \in C \exists$ a closed cube $C_{x} \times C_{x}^{\prime}$ where $C_{x}$ is a closed ball in $R^{q}$ and $C_{x}^{\prime}$ is a closed ball in $R^{n-q}$, such that $x \in\left(\right.$ Int $\left.C_{x}\right) \times\left(\right.$ Int $\left.C_{x}^{\prime}\right)$ and $C_{x} \times C_{x}^{\prime} \subset V$. Now the collection $\left\{\left(\right.\right.$ Int $\left.C_{x}\right) \times\left(\right.$ Int $\left.\left.C_{x}^{\prime}\right) / x \in C\right\}$ is an open cover of $C$ so $\exists$ a finite subcover, say $\left\{\left(\right.\right.$ Int $\left.C_{1}\right) \times\left(\right.$ Int $\left.C_{1}^{\prime}\right), \ldots,\left(\right.$ Int $\left.C_{r}\right) \times\left(\right.$ Int $\left.\left.C_{r}^{\prime}\right)\right\}$. Order the nonempty subsets $\left\{\alpha_{i}\right\}$ of $\{1, \ldots, r\}$ in such a way that $\alpha_{i} \subset \alpha_{j} \Rightarrow i \geqq j$. Let $s=2^{r}-1$. For all $i=1, \ldots, s$ define compact subsets $K_{i}$ of $R^{n-q}$ inductively as follows. $K_{1}$ should contain $\bigcap_{i=1}^{r} C_{i}^{\prime}$ in its interior and should satisfy $\left(\bigcup_{i=1}^{r} C_{i}\right) \times K_{1} \subset V$. Given $K_{1}, \ldots, K_{i-1}$, choose $K_{i}$ to satisfy:

(1) $K_{i} \cap K_{j} \neq \varnothing$ for $j<i \Leftrightarrow \alpha_{i} \subset \alpha_{j}$, in which case $K_{i} \cap K_{j}=\partial K_{i} \cap \partial K_{j}$;

(2) $\bigcup\left\{K_{j} / \alpha_{j} \supset \alpha_{i}\right\}$ contains $\bigcap_{j \in \alpha_{i}} C_{j}^{\prime}$ in its interior;

(3) $\left(\bigcup_{j \in \alpha_{i}} C_{j}\right) \times K_{i} \subset V$.

For convenience, we will abbreviate $\bigcup_{i=1}^{k}\left[\left(\bigcup_{j \in \alpha_{i}} C_{j}\right) \times K_{i}\right]$ to $U^{k}$. Using (2) one can show that

$$
C \subset \bigcup_{i=1}^{r} C_{i} \times C_{i}^{\prime} \subset U^{s} .
$$

Next, for all $i=1, \ldots, s$, choose open sets $U_{i}$ in $R^{n-q}$ satisfying

(1) $K_{i} \subset U_{i}$;

(2) if $\alpha_{i} \notin \alpha_{j}$ and $\alpha_{j} \notin \alpha_{i}$, then $U_{i} \cap U_{j}=\varnothing$;

(3) $\left(\bigcup_{j \in \alpha_{i}} C_{j}\right) \times\left(\mathrm{Cl} U_{i}\right) \subset V$. 
The deformation of the suitably chosen neighbourhood $\mathscr{P}$ will take $s$ steps, the $k$ th step of which will be a deformation of a neighbourhood $\mathscr{P}_{k}$ of $\eta$ in $\mathscr{E}_{\wp}\left(V, U^{k-1} ; R^{n}\right)$ down into $\mathscr{E}_{\mathfrak{\wp}}\left(V, U^{k} ; R^{n}\right)$, with the deformation taking place in $\mathscr{P}_{k}^{\prime}$.

Choose $\mathscr{P}_{s}^{\prime}$ to be a neighbourhood of $\eta$ in $\mathscr{E}_{\wp}\left(V, U^{s-1} ; R^{n}\right)$ such that $\mathscr{P}_{s}^{\prime} \subset \mathscr{Q}$.

Given a neighbourhood $\mathscr{P}_{k+1}^{\prime}$ of $\eta$ in $\mathscr{E}_{\wp}\left(V, U^{k} ; R^{n}\right)$ such that $\mathscr{P}_{k+1}^{\prime} \subset \mathscr{Q}$ choose $\mathscr{P}_{k+1}$ and $\mathscr{P}_{k}^{\prime}$ as follows. Apply Lemma 15 with $A=\bigcup_{j \in \alpha_{k+1}} C_{j}, B=K_{k+1}, D=U^{k}$, $W=U_{k+1}$ and $U$ a suitably small open neighbourhood of $A$ in $R^{q}$ chosen so that $(U \times W) \cap \bigcup\left\{\left(\bigcup_{j \in \alpha_{i}} C_{j}\right) \times K_{i} / \alpha_{i} \notin \alpha_{k+1}\right.$ and $\left.\alpha_{k+1} \notin \alpha_{i}\right\}=\varnothing$. Lemma 15 gives us a neighbourhood $\mathscr{P}_{k+1}$ of $\eta$ in $\mathscr{E}_{\wp}\left(V, U^{k} ; R^{n}\right)$ and a deformation of $\mathscr{P}_{k+1}$ in $\mathscr{P}_{k+1}^{\prime}$ down into $\mathscr{E}_{\wp}\left(V, U^{k+1} ; R^{n}\right)$. Now if $k>0$, choose a neighbourhood $\mathscr{P}_{k}^{\prime}$ of $\eta$ in $\mathscr{E}_{\wp}\left(V, U^{k-1} ; R^{n}\right)$ such that $\mathscr{P}_{k}^{\prime} \subset \mathscr{P}_{k+1}$.

Define the neighbourhood $\mathscr{P}$ to be $\mathscr{P}_{1}$. Then $\mathscr{P}$ is a neighbourhood of $\eta$ in $\mathscr{E}_{\mathfrak{p}}\left(V ; R^{n}\right)$. The above gives us a sequence of deformations of $\mathscr{P}$ in $\mathscr{Q}$ down into $\mathscr{E}_{\wp}\left(V, U^{s} ; R^{n}\right)$. But $\mathscr{E}_{\wp}\left(V, U^{s} ; R^{n}\right) \subset \mathscr{E}_{\wp}\left(V, C ; R^{n}\right)$, so that we have found the required neighbourhood and deformation.

Proof of Theorem 13. By Lemma 4(ii), for all $x \in N, \exists \varepsilon_{x}>0$ and local charts

$$
h: \varepsilon_{x} \tilde{I}^{r} \times R^{n} \rightarrow \varepsilon_{x} \tilde{I}^{r} \times N, \quad g: \varepsilon_{x} \tilde{I}^{r} \times R^{q} \rightarrow \varepsilon_{x} \tilde{I}^{r} \times Q,
$$

with $x \in p_{2} h\left(t \times R^{n}\right)$ for all $t \in \varepsilon_{x} \tilde{I}^{r}, h$ and $g$ commuting with projection onto $\varepsilon_{x} \tilde{I}^{r}$, such that if $(t, y) \in \varepsilon_{x} \tilde{I}^{r} \times R^{q}$, then $p_{2} g(t, y)=p_{2} g(0, y)$, and

$$
g^{-1} f h=1 \times 8: \varepsilon_{x} \tilde{I}^{r} \times R^{n} \rightarrow \varepsilon_{x} \tilde{I}^{r} \times R^{q}
$$

By compactness of $M$ we can cover $M$ by finitely many open sets $U_{i}$ where $U_{i}=h_{0, i}\left(R^{n}\right)$,

$$
h_{t, i}: R^{n} \rightarrow N \quad\left(t \in \varepsilon_{x_{i}} \tilde{I}^{r}\right)
$$

being the embedding defined by $h_{t, i}(y)=p_{2} h_{i}(t, y)$, where

$$
h_{i}: \varepsilon_{x_{i}} \tilde{I}^{r} \times R^{n} \rightarrow \varepsilon_{x_{i}} \tilde{I}^{r} \times N
$$

is the chart corresponding to $x_{i} \in N$.

Let $\varepsilon \leqq \min _{i} \varepsilon_{x_{i}}$. Refine $\left\{U_{i}\right\}$ to an open cover $\left\{V_{i}\right\}$ of $M$; each $V_{i}$ being open in $N$ and $\mathrm{Cl} V_{i} \subset U_{i}$. We may choose $\varepsilon>0$ small enough so that for all $t \in \varepsilon \tilde{I}^{r}$,

$$
M \subset \bigcup_{i} h_{t, i} h_{0, i}^{-1}\left(V_{i}\right) \text {. }
$$

We will inductively construct a cube

$$
\bar{H}_{t, i}: W_{i} \rightarrow N
$$

of embeddings, for $t \in \varepsilon \tilde{I}^{r}$, where $W_{i}$ is a closed neighbourhood of $\bigcup_{j=1}^{i}\left(\mathrm{Cl} V_{j}\right)$ in $N$, satisfying $\bar{H}_{0, i}=1$ and $f_{t} \bar{H}_{t, i}=f_{0} \mid W_{i}$, where $f_{t}$ is as before.

Start of the induction $(i=1)$. Choose $W_{1} \subset U_{1}$ such that $\mathrm{Cl} V_{1} \subset$ Int $W_{1}$ and set $\bar{H}_{t, 1}=h_{t, 1} h_{0,1}^{-1} \mid W_{1}$. Then $\bar{H}_{0,1}=1$ and $f_{t} \bar{H}_{t, 1}=f_{t} h_{t, 1} h_{0,1}^{-1}=g_{1} \wp h_{0,1}^{-1}=f_{0}$, where $g_{i}$ : $R^{q} \rightarrow Q$ is the embedding so that

$$
1 \times g_{i}: \varepsilon_{x_{i}} \tilde{I}^{r} \times R^{q} \rightarrow \varepsilon_{x_{i}} \tilde{I}^{r} \times Q
$$

is the $g$ given to us by Lemma 4(ii). 
Continuation of the induction. Suppose we are given $W_{i}$ and $\bar{H}_{t, i}$. We will construct $W_{i+1}$ and $\bar{H}_{t, i+1}$.

Let $X_{i+1}$ and $Y_{i+1}$ be open sets satisfying $\mathrm{Cl} V_{i+1} \subset Y_{i+1} \subset \mathrm{Cl} Y_{i+1} \subset X_{i+1}$ $\subset \mathrm{Cl} X_{i+1} \subset U_{i+1}$, and let $W_{i}^{\prime}$ and $W_{i}^{\prime \prime}$ be closed sets satisfying $\bigcup_{j=1}^{i} \mathrm{Cl} V_{j}$ $\subset$ Int $W_{i}^{\prime} \subset W_{i}^{\prime} \subset$ Int $W_{i}^{\prime \prime} \subset W_{i}^{\prime \prime} \subset$ Int $W_{i}$. Set $W_{i+1}=W_{i}^{\prime} \cup\left(\mathrm{Cl} Y_{i+1}\right)$. If $\varepsilon$ is chosen small enough, then $\bar{H}_{t, i}\left(W_{i} \cap \mathrm{Cl} X_{i+1}\right) \subset h_{t, i+1}\left(R^{n}\right)$.

Consider the open set $Z_{i}=h_{0, i+1}^{-1}\left(\right.$ Int $\left.W_{i} \cap X_{i+1}\right) \subset R^{n}$. The composition $h_{t, i+1}^{-1} \bar{H}_{t, i} h_{0, i+1}$ gives another embedding of $Z_{i}$ in $R^{n}$. Moreover, on $Z_{i}$, we have

$$
\wp=\wp h_{t, i+1}^{-1} \bar{H}_{t, i} h_{0, i+1},
$$

by use of the inductive hypothesis. Thus $h_{t, i+1}^{-1} \bar{H}_{t, i} h_{0, i+1} \mid Z_{i} \in \mathscr{E}_{\mathfrak{p}}\left(Z_{i} ; R^{n}\right)$. Note that when $t=0$, this embedding is just the inclusion, so if we let $C_{i}$ denote a compact set in $R^{n}$ containing $h_{0, i+1}^{-1}\left(\mathrm{Cl} Y_{i+1} \cap W_{i}^{\prime}\right)$ in its interior and contained in $Z_{i}^{\prime \prime}=h_{0, i+1}^{-1}$ (Int $\left.W_{i}^{\prime \prime} \cap X_{i+1}\right)$, Lemma 16 allows us to pick $\varepsilon$ so small that the cube of embeddings $\left\{h_{t, i+1}^{-1} \bar{H}_{t, i} h_{0, i+1} \mid Z_{i} / t \in \varepsilon \tilde{I}^{r}\right\}$ deforms into $\mathscr{E}_{\wp}\left(Z_{i}, C_{i} ; R^{n}\right)$. Moreover this deformation can be chosen to leave the cube alone on $Z_{i}-Z_{i}^{\prime \prime}$. Let the end product of the deformation of $h_{t, i+1}^{-1} \bar{H}_{t, i} h_{0, i+1} \mid Z_{i}$ be denoted by $\bar{h}_{t, i+1}: Z_{i} \rightarrow R^{n}$. Then

(1) $\bar{h}_{t, i+1}=h_{t, i+1}^{-1} \bar{H}_{t, i} h_{0, i+1}$ on $Z_{i}-Z_{i}^{\prime \prime}$;

(2) $\bar{h}_{t, i+1}=1$ on $C_{i}$;

(3) $\wp=\wp \bar{h}_{t, i+1}$;

(4) $\bar{h}_{0, i+1}=1$;

(5) the embeddings $\bar{h}_{t, i+1}$ vary continuously with respect to $t$.

Now $W_{i}^{\prime \prime} \cap \mathrm{Cl} Y_{i+1}$-Int $W_{i}^{\prime}$ is a closed set contained in the open set Int $W_{i}$ $\cap X_{i+1}$. Hence by (4) and (5), if $\varepsilon$ is small enough, we have

$$
h_{0, i+1}^{-1}\left(W_{i}^{\prime \prime} \cap \mathrm{Cl} Y_{i+1}-\text { Int } W_{i}^{\prime}\right) \subset \bar{h}_{t, i+1}\left(Z_{i}\right) \text {. }
$$

Define $\bar{H}_{t, i+1}$ as follows. Let $x \in W_{i+1}$. Then

$$
\begin{aligned}
\bar{H}_{t, i+1}(x) & =\bar{H}_{t, i}(x) & & \text { if } x \in W_{i}^{\prime}, \\
& =\bar{H}_{t, i} h_{0, i+1} \bar{h}_{t, i+1}^{-1} h_{0, i+1}^{-1}(x) & & \text { if } x \in W_{i}^{\prime \prime} \cap \mathrm{Cl} Y_{i+1}-\text { Int } W_{i}^{\prime}, \\
& =h_{t, i+1} h_{0, i+1}^{-1}(x) & & \text { if } x \in \mathrm{Cl} Y_{i+1}-\text { Int } W_{i}^{\prime \prime} .
\end{aligned}
$$

One can readily check that the union of the above three subdomains is $W_{i+1}$. One can also check that $\bar{H}_{t, i+1}$ is a well-defined mersion (hence an embedding for sufficiently small $\varepsilon$ ), that $\bar{H}_{0, i+1}=1$ and that $f_{t} \bar{H}_{t, i+1}=f_{0} \mid W_{i+1}$.

This completes the inductive construction of the cube $\bar{H}_{t, i}$. We thus have a cube of embeddings

$$
\bar{H}_{t}: W \rightarrow N \quad\left(t \in \varepsilon \tilde{I}^{r}\right),
$$

where $W$ is a closed neighbourhood of $M$ in $N$, satisfying $\bar{H}_{0}=1$ and $f_{t} \bar{H}_{t}=f_{0} \mid W$. Since only a finite number of adjustments were made to $\varepsilon$, we still have $\varepsilon>0$. By continuity of the cube with respect to $t$ and since $\bar{H}_{0}=1$, we may assume $\varepsilon>0$ so small that $M \subset \bar{H}_{t}(W)$. Now define $H_{t}: M \rightarrow N$ by $\bar{H}_{t}(x)=H_{t}^{-1}(x)$. If $\varepsilon$ is small enough then $H_{t}(M) \cap \partial N=\varnothing$. Now extend the isotopy $\left\{H_{t}\right\}$ above to a cube of 
homeomorphisms $H_{t}: N \rightarrow N$ so that $H_{t} \mid \partial N=1$, using the Isotopy Extension Theorem of Edwards and Kirby [2]. The required homeomorphism is then given by $H(t, x)=\left(t, H_{t}(x)\right)$.

7. Proof of the fibration lemma for mersions. It suffices to consider the case where $M-M_{0}$ is a single $k$-handle, $k<n$, as this then enables us to prove the result by induction. We thus have the following lifting problem:

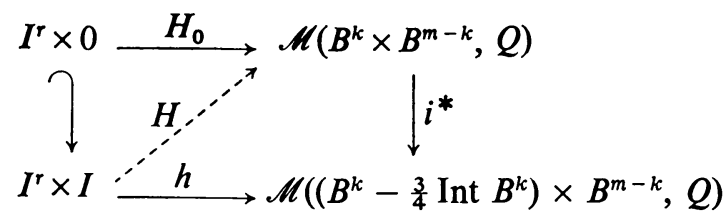

By Lemma 3, $h$ may be thought of as a cube, $h_{t, s}:\left(3 B^{k}-\frac{1}{4}\right.$ Int $\left.B^{k}\right) \times 4 B^{n-k}$ $\rightarrow Q\left((t, s) \in I^{r} \times I\right)$, of mersions, where we take $\left(3 B^{k}-\frac{1}{4}\right.$ Int $\left.B^{k}\right) \times 4 B^{n-k}$ to be the abstract regular neighbourhood of $\left(B^{k}-\frac{3}{4}\right.$ Int $\left.B^{k}\right) \times B^{m-k}$ furnished by the definition of $\mathscr{M}_{\theta}(M, Q)$. Similarly $H$ may be thought of as a cube

$$
H_{t, s}: 3 B^{k} \times 4 B^{n-k} \rightarrow Q \quad\left((t, s) \in I^{r} \times I\right)
$$

of mersions. Our task is to extend $h_{t, s} \mid\left(2 B^{k}-\frac{2}{3}\right.$ Int $\left.B^{k}\right) \times 2 B^{n-k}$ to $H_{t, s}$ given $H_{t, 0}$. We may assume that

$$
h_{t, 0} \mid\left(2 B^{k}-\frac{1}{3} \text { Int } B^{k}\right) \times 2 B^{n-k}=H_{t, 0} \mid\left(2 B^{k}-\frac{1}{3} \text { Int } B^{k}\right) \times 2 B^{n-k},
$$

by commutativity of the above square.

The major difficulty to be overcome is illustrated by the following example, which also shows the necessity of the hypothesis $k<n$.

EXAMPLE. Take $r=0, m=k, q=2, Q=R^{2}$. Let $H_{0}$ be the standard embedding of $3 B^{2}$ in $R^{2}$ and $h_{s}$ be the regular homotopy which fixes points below the $x$-axis and drags $(0,1)$ linearly along the $y$-axis to $(0,-1)$, see Figure 2 . We see that there will be no difficulty extending $h_{s} \mid\left(2 B^{2}-\frac{2}{3}\right.$ Int $\left.B^{2}\right)$ to $H_{s}$ when $s<\frac{2}{3}$, but $h_{2 / 3}$ cannot even be extended to a mersion let alone one which would make $H_{s}$ a regular homotopy.

The above example can be adapted to give a regular homotopy of submersions of an open annulus in $R^{1}$ : merely take the interior of the above annulus and project the various stages in Figure 2 onto the $y$-axis. Again there will be trouble when $s=2 / 3$.

We precede the proof of Lemma 9 by an outline of it.

Using the compactness of $I^{r} \times I$ and Theorem 13 above, we will reduce our consideration to the case where Condition $(*)$ is satisfied.

Condition (*). $\exists t_{0} \in I^{r}$ such that if $(t, s) \in I^{r} \times I$, then

$$
h_{t, s}\left[\left(2 B^{k}-\frac{1}{3} \operatorname{Int} B^{k}\right) \times 2 B^{n-k}\right] \subset h_{t_{0}, 0}\left[\left(3 \text { Int } B^{k}-\frac{1}{4} B^{k}\right) \times 3 \text { Int } B^{n-k}\right],
$$



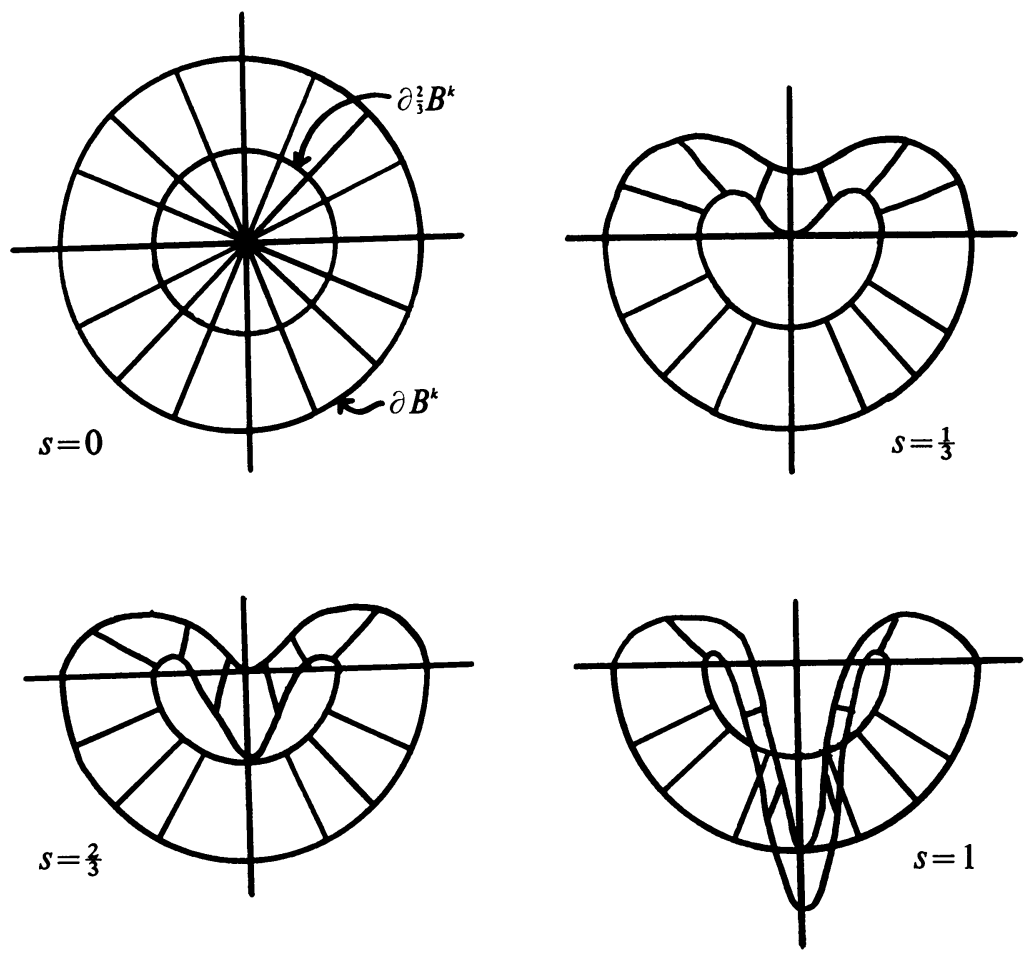

FIGURE 2

and $\exists$ a cube

$$
g_{t, s}:\left(2 B^{k}-\frac{1}{3} \text { Int } B^{k}\right) \times 2 B^{n-k} \rightarrow\left(3 \text { Int } B^{k}-\frac{1}{4} B^{k}\right) \times 3 \text { Int } B^{n-k}
$$

of embeddings such that $g_{t_{0}, 0}=1$ and for all $(t, s) \in I^{r} \times I$,

$$
h_{t_{0}, 0} g_{t, s}=h_{t, s}\left(2 B^{k}-\frac{1}{3} \text { Int } B^{k}\right) \times 2 B^{n-k} \text {. }
$$

We will assume $t_{0}=0$.

Condition (*) says that the cube $h_{t, s}$ of mersions can be realised by composing a cube of embeddings in $M$ with the mersion $h_{0,0}$. Thereafter we will work with the cube $g_{t, s}$ of embeddings rather than the mersions $h_{t, s}$. We will define $H_{t, s}$ to be $H_{t, 0}$ on $\frac{1}{3} B^{k} \times 2 B^{n-k}$. We would like to be able to define $H_{t, s}$ on

$$
\left(\frac{2}{3} B^{k}-\frac{1}{3} \text { Int } B^{k}\right) \times 2 B^{n-k}
$$

to be just $H_{t, 0} g_{t, s}$, but this does not overcome the difficulty of the example above. It is here that the hypothesis $k<n$ comes to our rescue, for in this case the factor $4 B^{n-k}$ contains a line. Lemma 18 below will allow us to "push $\frac{1}{2} \partial B^{k} \times 4 B^{n-k}$ out of the way" along this line during the interval $s \in[0, \varepsilon]$. This would correspond to lifting a circle in the annulus of Figure 2 up out of the plane. While this push is being carried out, we essentially use $g_{t, s}$ to extend $H_{t, s}(s \leqq \varepsilon)$ over $\left(\frac{2}{3} B^{k}-\frac{1}{3} \operatorname{Int} B^{k}\right)$ $\times 2 B^{n-k}$. $H_{t, \varepsilon}$ will then be in the "good position" of Haefliger and Poenaru; in fact we will have pushed $\frac{1}{2} \partial B^{k} \times 2 B^{n-k}$ out into $2 B^{k} \times\left(4 B^{n-k}-3 B^{n-k}\right)$. For $s \geqq \varepsilon$, 
we will define $H_{t, s}$ to be $H_{t, \varepsilon}$ on $\left(\frac{1}{2} B^{k}-\frac{1}{3} \operatorname{Int} B^{k}\right) \times 2 B^{n-k}$ and on $\left(\frac{2}{3} B^{k}-\frac{1}{2} \operatorname{Int} B^{k}\right)$ $\times 2 B^{n-k}$ we will essentially just extend $g_{t, s}$ to a cube of homeomorphisms on $3 B^{k} \times 3 B^{n-k}$ which fix the boundary and then see what the cube does to $H_{t, \varepsilon}$.

Proof that the truth of Lemma 9 under Condition (*) implies Lemma 9 in the general case. For all $(t, s) \in I^{r} \times I$, choose a basic closed cube $I_{t, s}$, a neighbourhood of $(t, s)$ in $I^{r} \times I$, as follows. Since the closed set $\left(2 B^{k}-\frac{1}{3}\right.$ Int $\left.B^{k}\right) \times 2 B^{n-k}$ is contained in the open set ( 3 Int $\left.B^{k}-\frac{1}{4} B^{k}\right) \times 3$ Int $B^{n-k}$, we can choose $I_{t, s}$ so small that if $(\tau, \sigma)$ and $\left(\tau^{\prime}, \sigma^{\prime}\right) \in I_{t, s}$, then

$$
h_{\tau, \sigma}\left[\left(2 B^{k}-\frac{1}{3} \operatorname{Int} B^{k}\right) \times 2 B^{n-k}\right] \subset h_{\tau^{\prime}, \sigma^{\prime}}\left[\left(3 \text { Int } B^{k}-\frac{1}{4} B^{k}\right) \times 3 \text { Int } B^{n-k}\right] .
$$

Let $V$ and $W$ be two compact submanifolds of the interior of $\left(3 B^{k}-\frac{1}{4} B^{k}\right) \times 3 B^{n-k}$ such that

$$
\left(2 B^{k}-\frac{1}{3} \text { Int } B^{k}\right) \times 2 B^{n-k} \subset \text { Int } V \subset V \subset \text { Int } W .
$$

Then by Theorem 13, if $I_{t, s}$ is small enough, we can find a cube of homeomorphisms $g_{t, s, \tau, \sigma}:\left(3 B^{k}-\frac{1}{4}\right.$ Int $\left.B^{k}\right) \times 3 B^{n-k} \rightarrow\left(3 B^{k}-\frac{1}{4}\right.$ Int $\left.B^{k}\right) \times 3 B^{n-k}$ such that $g_{t, s, t, s}=1$, $g_{t, s, \tau, \sigma} \mid \partial\left[\left(3 B^{k}-\frac{1}{4}\right.\right.$ Int $\left.\left.B^{k}\right) \times 3 B^{n-k}\right]=1, h_{t, s} g_{t, s, \tau, \sigma}=h_{\tau, \sigma}$ on $W$, and

$$
g_{t, s, \tau, \sigma}\left[\left(2 B^{k}-\frac{1}{3} \operatorname{Int} B^{k}\right) \times 2 B^{n-k}\right] \subset V \subset g_{t, s, \tau, \sigma}(W),
$$

for all $(\tau, \sigma) \in I_{t, s}$.

Now the collection $\left\{\right.$ Int $\left.I_{t, s} /(t, s) \in I^{r} \times I\right\}$ is an open cover of $I^{r} \times I$. Let $\left\{I_{1}, \ldots, I_{k}\right\}$ denote the closures of the members of a finite subcover. Let $\left\{J_{1}, \ldots, J_{l}\right\}$ denote another cover given to us by Lemma 5 . Let $K_{j}$ be as in Lemma 5. Extend $h_{t, s}$ to $H_{t, s}$ over each of the cubes $J_{j}$ in order thus: suppose $h_{t, s}$ has been extended to $H_{t, s}$ for $(t, s) \in K_{j}(j \geqq 1)$. Since $J_{j} \cap K_{j}$ is an $r$-ball in $\partial J_{j}, J_{j}$ can be thought of as a homeomorphism of $I^{r} \times I$ with $J_{j} \cap K_{j}$ as the "floor" $I^{r} \times 0$. It suffices to verify Condition (*). Let $(t, s) \in J_{j} \cap K_{j}$ denote the point corresponding to $(0,0)$ under the above identification. $\exists i$ so that $J_{j} \subset I_{i}$, and $I_{i}$ was the neighbourhood corresponding to $\left(t_{i}, s_{i}\right)$ in the cube. Then

$$
h_{\tau, \sigma}\left[\left(2 B^{k}-\frac{1}{3} \text { Int } B^{k}\right) \times 2 B^{n-k}\right] \subset h_{t, s}\left[\left(3 \text { Int } B^{k}-\frac{1}{4} B^{k}\right) \times 3 \text { Int } B^{n-k}\right]
$$

for all $(\tau, \sigma) \in J_{j} \subset I_{i}$, so that the first part of Condition $(*)$ is satisfied. One may check that the remaining parts are satisfied if the cube of homeomorphisms

$$
g_{\tau, \sigma}:\left(3 B^{k}-\frac{1}{4} \text { Int } B^{k}\right) \times 3 B^{n-k} \rightarrow\left(3 B^{k}-\frac{1}{4} \text { Int } B^{k}\right) \times 3 B^{n-k}
$$

is defined by $g_{\tau, \sigma}=g_{t_{i}, s_{i}, t, s}^{-1} g_{t_{i}, s_{i}, \tau, \sigma}$.

We will therefore assume Condition (*).

LEMMA 17. Let $M^{m}$ be a manifold with $C$ a compact subset of $M$ so that $C \cap \partial M$ $=\varnothing$, and $U$ a neighbourhood of $C$ in $M$. Suppose given a cube $\alpha_{t, s}: U \rightarrow M$ of embeddings of $U$ in $M,(t, s) \in I^{r} \times I$, and a cube $\beta_{t, 0}: M \rightarrow M$ of homeomorphisms such that $\beta_{t, 0} \mid \partial M=1$ and $\alpha_{t, 0}=\beta_{t, 0} \mid C$. Then we can extend the cube $\beta_{t, 0}$ to a cube $\beta_{t, s}$ : $M \rightarrow M$ of homeomorphisms such that $\beta_{t, s} \mid \partial M=1$ and $\alpha_{t, s}=\beta_{t, s} \mid C$. 
Proof. By the Isotopy Extension Theorem, Edwards and Kirby [2] we can extend $\alpha_{t, s} \mid C$ to a cube $\gamma_{t, s}: M \rightarrow M$ of homeomorphisms such that $\gamma_{t, s} \mid \partial M=1$. Define the required $\beta_{t, s}$ by $\beta_{t, s}=\gamma_{t, s} \gamma_{t, 0}^{-1} \beta_{t, 0}$.

Lemma 18 (CF. Haefliger ANd Poenaru). Under Condition (*), $\exists \varepsilon>0$, an arbitrarily small neighbourhood $U$ of $\left(2 B^{k}-\frac{2}{3}\right.$ Int $\left.B^{k}\right) \times 2 B^{n-k}$ in $2 B^{k} \times 2 B^{n-k}$ and a cube

$$
f_{t, s}:\left(3 B^{k}-\frac{1}{4} \text { Int } B^{k}\right) \times 3 B^{n-k} \rightarrow\left(3 B^{k}-\frac{1}{4} \operatorname{Int} B^{k}\right) \times 3 B^{n-k}
$$

of homeomorphisms, $(t, s) \in I^{r} \times[0, \varepsilon]$, fixing $\partial\left[\left(3 B^{k}-\frac{1}{4}\right.\right.$ Int $\left.\left.B^{k}\right) \times 3 B^{n-k}\right]$ satisfying:

(i) $f_{t, s}=g_{t, s}$ on $\left(2 B^{k}-\frac{2}{3}\right.$ Int $\left.B^{k}\right) \times 2 B^{n-k}$;

(ii) $h_{0,0} f_{t, 0}\left|U=H_{t, 0}\right| U$;

(iii) $f_{t, s}=f_{t, 0}$ on the frontier of $U$ in $2 B^{k} \times 2 B^{n-k}$.

Proof. Take $U$ to be any neighbourhood of $\left(2 B^{k}-\frac{2}{3}\right.$ Int $\left.B^{k}\right) \times 2 B^{n-k}$ in $\left(2 B^{k}-\frac{1}{3} B^{k}\right)$ $\times 2 B^{n-k}$ such that the closure of $U$ is a submanifold with boundary. Now $g_{t, 0}$ is defined on $U$ and is a cube of embeddings of $U$ in ( 3 Int $\left.B^{k}-\frac{1}{4} B^{k}\right) \times 3$ Int $B^{n-k}$. Thus by the Isotopy Extension Theorem, $\exists$ a cube of homeomorphisms

$$
\gamma_{t}:\left(3 B^{k}-\frac{1}{4} \text { Int } B^{k}\right) \times 3 B^{n-k} \rightarrow\left(3 B^{k}-\frac{1}{4} \text { Int } B^{k}\right) \times 3 B^{n-k}
$$

such that $\gamma_{t}=g_{t, 0}$ on $U$ and $\gamma_{t}=1$ on the boundary.

Now let $V$ be a compact manifold in the interior of $\left(3 B^{k}-\frac{1}{4} B^{k}\right) \times 3 B^{n-k}$ which is a neighbourhood of $\left(2 B^{k}-\frac{2}{3}\right.$ Int $\left.B^{k}\right) \times 2 B^{n-k}$ containing $U$, with the frontier of $U$ in $2 B^{k} \times 2 B^{n-k}$ contained in $\partial V$. Now on $\left(2 B^{k}-\frac{2}{3}\right.$ Int $\left.B^{k}\right) \times 2 B^{n-k}, \gamma_{t}^{-1} g_{t, 0}=1$. Hence $\exists \varepsilon>0$ so that $\gamma_{t}^{-1} g_{t ; s}\left[\left(2 B^{k}-\frac{2}{3}\right.\right.$ Int $\left.\left.B^{k}\right) \times 2 B^{n-k}\right] \subset V$ when $s \leqq \varepsilon$. Thus we may apply Lemma 17 to extend $1_{V}$ and $\gamma_{t}^{-1} g_{t, s} \mid\left(2 B^{k}-\frac{2}{3}\right.$ Int $\left.B^{k}\right) \times 2 B^{n-k}$ to a cube of homeomorphisms $\delta_{t, s}: V \rightarrow V, s \leqq \varepsilon$, with $\delta_{t, s} \mid \partial V=1$. Finally, define $f_{t, s}(s \leqq \varepsilon)$ by

$$
\begin{aligned}
f_{t, s}(x) & =\gamma_{t} \delta_{t, s}(x) & & \text { if } x \in V, \\
& =\gamma_{t}(x) & & \text { if } x \notin V .
\end{aligned}
$$

REMARK 1. Since $f_{t, s} \mid \partial=1$, we can extend $f_{t, s}$ to a cube of homeomorphisms on $\left(3 B^{k}-\frac{1}{4}\right.$ Int $\left.B^{k}\right) \times 4 B^{n-k}$, by defining them to be 1 on the added domain. We will assume $f_{t, s}$ to be so extended.

REMARK 2. For simplicity of notation, we may take $U=\left(2 B^{k}-\frac{1}{3}\right.$ Int $\left.B^{k}\right) \times 2 B^{n-k}$. The "pushing" of $\frac{1}{2} \partial B^{k} \times 2 B^{n-k}$ requires the map

defined by

$$
\alpha:[0, \varepsilon] \times\left[\frac{1}{3}, 2\right] \times[-2,2] \rightarrow[-2,4]
$$

$$
\begin{aligned}
\alpha(s, x, y) & =y+(1-3|2 x-1|) \frac{s}{\varepsilon}\left(\frac{7}{2}-\frac{3 y}{4}\right) & & \text { if } x \leqq \frac{2}{3}, \\
& =y & & \text { if } x \geqq \frac{2}{3} .
\end{aligned}
$$

Note that $\alpha(0, x, y)=\alpha\left(x, \frac{2}{3}, y\right)=\alpha\left(s, \frac{1}{3}, y\right)=y$ and $\alpha \mid\{\varepsilon\} \times\left\{\frac{1}{2}\right\} \times[-2,2]$ takes $[-2,2]$ linearly to $[3,4]$. 
Thinking of $B^{l}$ as the $l$-fold product of $[-1,1]$ and letting $|x|=\max \left\{\left|x_{1}\right|, \ldots,\left|x_{l}\right|\right\}$ when $x=\left(x_{1}, \ldots, x_{l}\right) \in B^{l}$, we may define

$$
\beta:[0, \varepsilon] \times\left(2 B^{k}-\frac{1}{3} \text { Int } B^{k}\right) \times 2 B^{n-k} \rightarrow\left(2 B^{k}-\frac{1}{3} \text { Int } B^{k}\right) \times 4 B^{n-k}
$$

by $\beta\left(s, x,\left(y_{1}, \ldots, y_{n-k}\right)\right)=\left(x,\left(\alpha\left(s,|x|, y_{1}\right), y_{2}, \ldots, y_{n-k}\right)\right)$. It is here that we require the hypothesis $k<n$.

$H_{t, s}$ may now be defined for $s \leqq \varepsilon$ as follows:

$$
\begin{aligned}
H_{t, s}(z) & =h_{0,0} f_{t, s} \beta(s, z) & & \text { if } z \in\left(2 B^{k}-\frac{1}{3} \text { Int } B^{k}\right) \times 2 B^{n-k}, \\
& =H_{t, 0}(z) & & \text { if } z \in \frac{1}{3} B^{k} \times 2 B^{n-k} .
\end{aligned}
$$

One can readily check that $H_{t, s}$ so defined is well-defined and is a regular homotopy.

Now that we have put $H_{t, \varepsilon}$ into a "good position", we can extend to $H_{t, s}(s \geqq \varepsilon)$ as in Haefliger and Poenaru. The Isotopy Extension Theorem allows us to extend the isotopy $\left\{g_{t, s}\right\}$ given by Condition $(*)$ to a cube of homeomorphisms $\left\{G_{t, s}\right\}$ of $\left(3 B^{k}-\frac{1}{4}\right.$ Int $\left.B^{k}\right) \times 4 B^{n-k}$ such that $G_{t, s} \mid\left(3 B^{k}-\frac{1}{4}\right.$ Int $\left.B^{k}\right) \times\left(4 B^{n-k}-3 B^{n-k}\right)=1$. Define

$$
\begin{aligned}
H_{t, s}(z) & =h_{0,0} G_{t, s} \beta(\varepsilon, z) & & \text { if } z \in\left(2 B^{k}-\frac{1}{2} \text { Int } B^{k}\right) \times 2 B^{n-k}, \\
& =H_{t, \varepsilon}(z) & & \text { if } z \in \frac{1}{2} B^{k} \times 2 B^{n-k} .
\end{aligned}
$$

It is readily checked that $H_{t, s}$ so defined satisfies the conditions required of it. This completes the proof of Lemma 9.

8. Proof of Theorem 1 in the general case. We start with an outline of the proof. If $\mathrm{Cl}(M-N)$ is compact, then we can cover it by finitely many coordinate patches. Each of these patches can be given a handlebody structure, but those structures on overlapping patches may not agree: this is why we cannot use the handlebody case. Instead, we apply the relative handlebody case to each coordinate patch in turn. If $\mathrm{Cl}(M-N)$ is not compact, we divide it into a countable increasing union of compact sets such that any set is contained in the interior of its successors, and then apply the previous case to each of these in turn.

On setting $K=S^{p}, L=\varnothing$, respectively $K=B^{p+1}, L=S^{p}$ in the following lemma, we get that

$$
d_{*}: \pi_{p}\left(\mathscr{M}_{\theta}(M, Q)\right) \rightarrow \pi_{p}\left(\mathscr{R}_{\theta}(M, Q)\right)
$$

is surjective, respectively injective. Thus by Theorem 12.5 in May [7], $d$ is a homotopy equivalence. In the lemma, we assume $M, N, M^{\prime}, Q$ and $\theta$ are as in Theorem 1.

LEMMA 19. Suppose given a finite simplicial complex $K$, a subcomplex $L$ and simplicial maps $\alpha: L \rightarrow \mathscr{M}_{\theta}(M, Q)$ and $\beta: K \rightarrow \mathscr{R}_{\theta}(M, Q)$ so that $d \alpha \cong \beta \mid L$. Then we can extend $\alpha$ to $\gamma: K \rightarrow \mathscr{M}_{\theta}(M, Q)$ such that $d \gamma \cong \beta$.

Proof. It suffices to consider the case where $K$ is a $p$-ball $B^{p}$ and $L$ is its boundary $S^{p-1}, p \geqq 0\left(S^{-1}=\varnothing\right)$, as this will give us an inductive method for extending $\alpha$ over the $p$-skeleton of $K-L$. 
By Lemma 3, $\alpha$ and $\beta$ correspond respectively to $f: S^{p-1} \times U \rightarrow S^{p-1} \times Q$ and $g: B^{p} \times W \rightarrow B^{p} \times Q \times Q, f$ and $g$ satisfying the usual conditions, $U$ a neighbourhood of $M$ in $M^{\prime}$ and $W$ a neighbourhood of $\Delta(U)$ in $M^{\prime} \times M^{\prime}$. Since $d \alpha \cong \beta \mid S^{p-1}$, we may assume that $U$ and $W$ are so small that $d f \cong g$ over $S^{p-1}$, say by $g_{t}$, with $g_{0}=g$, $g_{1}=d f$. Our task is to extend $f$ and $\theta$ to a mersion $\bar{f}: B^{p} \times V \rightarrow B^{p} \times Q, V$ a possibly smalle' neighbourhood of $M$ in $M^{\prime}$ and $\bar{f}$ satisfying the usual properties, with $d \bar{f} \cong g$.

Case I. Suppose firstly that $\mathrm{Cl}(M-N)$ is compact. Let $\left\{U_{i}\right\}$ be a finite cover of $\mathrm{Cl}(M-N)$ by open sets, each homeomorphic to $R^{n}$, and $U_{i} \subset U$. Refine $\left\{U_{i}\right\}$ to another open cover $\left\{V_{i}\right\}$ with $\mathrm{Cl} V_{i} \subset U_{i}$. Assume $\mathrm{Cl} V_{i}$ is compact. We will inductively construct maps

$$
\hat{f}_{i}: B^{p} \times \hat{W}_{i} \rightarrow B^{p} \times Q
$$

satisfying the usual conditions, with $\hat{W}_{i} \subset U$ and with $W_{i}=\hat{W}_{i} \cup P_{i}$ being a neighbourhood of $\bigcup_{j \leqq i}\left(\mathrm{Cl} V_{j}\right) \cup N$ for some finite set $P_{i} . \hat{f}_{i}$ will agree with $f$ on $S^{p-1}$ $\times \hat{W}_{i}$, and $d \hat{f}_{i} \cong g$ by a homotopy $\hat{g}_{t, i}$ such that $\hat{g}_{0, i}=g$ and $\hat{g}_{1, i}=d \hat{f}_{i}$ over $\hat{W}_{i}$.

Start of the induction. The induction starts trivially at $i=0$.

Continuation of the induction. Suppose $\hat{f}_{i-1}, W_{i-1}$ and $P_{i-1}$ have already been constructed. Give $U_{i}$ a handlebody structure in such a way that every handle meeting a neighbourhood of $\left(\mathrm{Cl} V_{i}\right) \cap\left[\bigcup_{j<i}\left(\mathrm{Cl} V_{j}\right) \cup N\right]$ lies in Int $W_{i-1}$, that these handles are chosen first and these are then followed by handles meeting $\left(\mathrm{Cl} V_{i}\right)-\left[\cup_{j<i}\left(\mathrm{Cl} V_{j}\right) \cup N\right]$. Let $\tilde{X}_{i}$ denote the union of the first handles above. Now $\tilde{X}_{i}$ is a manifold with boundary and is a neighbourhood of

$$
\left(\mathrm{Cl} V_{i}\right) \cap\left[\bigcup_{j<i}\left(\mathrm{Cl} V_{j}\right) \cup N\right]
$$

Hence by Brown [1, Theorem 2], $\partial \mathscr{X}_{i}$ is collared. Thus we can find another handlebody $\tilde{X}_{i}^{\prime}$ such that $\tilde{X}_{i}^{\prime}$ is also a neighbourhood of $\left(\mathrm{Cl} V_{i}\right) \cap\left[\bigcup_{j<i}\left(\mathrm{Cl} V_{j}\right) \cup N\right]$, $\tilde{X}_{i}^{\prime}$ is abstractly the same handlebody as $\tilde{X}_{i}$ and $\tilde{X}_{i}=\tilde{X}_{i}^{\prime} \cup \partial \tilde{X}_{i}^{\prime} \times[0,2]$, with $x \in \partial \tilde{X}_{i}^{\prime}$ identified with $(x, 0) \in \partial \tilde{X}_{i}^{\prime} \times[0,2]$. To obtain $\tilde{X}_{i}^{\prime}$, merely push $\tilde{X}_{i}$ along the collar of $\partial \tilde{X}_{i}$ a bit. Now alter the handlebody structure on $U_{i}$ a bit so that $\tilde{X}_{i}^{\prime}$ is a subhandlebody instead of $X_{i}$. Let $\hat{X}_{i}$ denote the union of those handles which either lie in $\tilde{X}_{i}^{\prime}$ or meet $\mathrm{Cl} V_{i}$ and have index $<n$. Let $X_{i}$ denote the union of all handles, in this new structure, which meet $\mathrm{Cl} V_{i}$. We will define $\hat{f}_{i}$ on $\hat{X}_{i}$, there being a natural extension over all of $X_{i}$ minus the centres of the $n$-handles. $P_{i}$ will then be the union of $P_{i-1}$ with the centres of the above $n$-handles.

Define a new representation over $U_{i}$,

$$
g^{\prime}: B^{p} \times\left[W \cap\left(U_{i} \times U_{i}\right)\right] \rightarrow B^{p} \times Q \times Q
$$

as follows: Over $U_{i}-\tilde{X}_{i}$, set $g^{\prime}=g$. Over $\tilde{X}_{i}^{\prime} \cup \partial \hat{X}_{i}^{\prime} \times[0,1]$, set $g^{\prime}=d \hat{f}_{i-1}$, and if $(y, t) \in \partial \tilde{X}_{i}^{\prime} \times[1,2], x \in B^{p}$ and $z \in M^{\prime}$ are such that

$$
(x,(y, t), z) \in B^{p} \times\left[W \cap\left(U_{i} \times U_{i}\right)\right],
$$


set $g^{\prime}(x,(y, t), z)=\hat{g}_{2-t, i-1}(x,(y, t), z)$. Define the homotopy $g_{s}^{\prime}$ over $S^{p-1}$ with $g_{0}^{\prime}=g^{\prime}$ and $g_{1}^{\prime}=d f, g_{s}^{\prime}$ constantly $d f=d \hat{f}_{i-1}$ over $S^{p-1} \times\left[\tilde{X}_{i}^{\prime} \cup \partial \tilde{X}_{i}^{\prime} \times[0,1]-P_{i-1}\right]$ thus: $g_{s}^{\prime}=g_{s}$ over $U_{i}-\tilde{X}_{i}$, and if $(x,(y, t), z)$ is as above, then

$$
g_{s}^{\prime}(x,(y, t), z)=\hat{g}_{(2-t)(1-s)+s, i-1}(x,(y, t), z) .
$$

Now $f$ and $g^{\prime}$ determine maps $\alpha_{i}$ and $\beta_{i}$ from $S^{p-1}$ and $B^{p}$ to $\mathscr{M}_{f_{i-1}}\left(\hat{X}_{i}-P_{i-1}, Q\right)$ and $\mathscr{R}_{f_{i-1}}\left(\hat{X}_{i}-P_{i-1}, Q\right)$ respectively, $g_{s}^{\prime}$ giving a homotopy between $d \alpha_{i}$ and $\beta_{i} \mid S^{p-1}$. By using that part of Theorem 1 already proven, we can extend $\alpha_{i}$ to

$$
\gamma_{i}: B^{p} \rightarrow \mathscr{M}_{f_{i-1}}\left(\hat{X}_{i}-P_{i-1}, Q\right)
$$

so that $d \gamma_{i} \simeq \beta_{i}$. Lemma 3 now provides a mersion

$$
\hat{f}_{i}: B^{p} \times W_{i}^{\prime} \rightarrow B^{p} \times Q
$$

satisfying the usual conditions and a homotopy from $g^{\prime}$ to $d \hat{f}_{i}$. Moreover, $\hat{f}_{i}$ and the homotopy agree with $\hat{f}_{i-1}$ on a neighbourhood of $\tilde{X}_{i}^{\prime}-P_{i-1}$, so that they extend over a neighbourhood $\hat{W}_{i}$ of $\left[N \cup \cup_{j<i}\left(\mathrm{Cl} V_{j}\right) \cup \hat{X}_{i}\right]-P_{i-1}$. Clearly $g$ is homotopic to $g^{\prime}$ by a homotopy which leaves $g$ alone over $U_{i}-\tilde{X}_{i}$. Thus $g \cong d \hat{f}_{i}$ over $U_{i}$ and hence over $\hat{W}_{i}$. This completes the induction, so we have a map $\hat{f}: B^{p} \times \hat{V} \rightarrow B^{p} \times Q$ satisfying the usual conditions, where $\hat{V} \cup P$ is a neighbourhood of $M$ in $M^{\prime}$ for some finite set $P$, and a homotopy $\hat{g}_{t}$ from $g$ to $d \hat{f}$. We may actually assume that $f$ and $g_{t}$ are defined over $B^{p}-\frac{1}{2}$ Int $B^{p}$ rather than just $S^{p-1}$ by a natural radial extension, and that $\hat{f}$ agrees with $f$ there.

After deleting points of $P$ as necessary, $P \subset \mathrm{Cl}(M-N)-N$. Run disjoint arcs through $M-N$ out into $M^{\prime}-M$ such that each point of $P$ lies on one of these arcs. These arcs have trivial normal bundles which may also be taken to be disjoint and to miss $N$. Now define an isotopy $h_{t}: V \rightarrow V$, where $V=\hat{V} \cup P$ as follows: $h_{0}=1, h_{1}(V) \subset \hat{V}, h_{t}=1$ off the union of the above bundles, and $h_{t}$ pushes along the normal bundles. Now define $\bar{f}: B^{p} \times V \rightarrow B^{p} \times Q$ by

$$
\begin{aligned}
\bar{f}(x, y) & =\hat{f}\left(x, h_{2(1-|x|)}(y)\right) & & \text { if }|x| \geqq \frac{1}{2}, \\
& =\hat{f}\left(x, h_{1}(y)\right) & & \text { if }|x| \leqq \frac{1}{2} .
\end{aligned}
$$

Note that if $x \in S^{p-1}$, then $\bar{f}(x, y)=\hat{f}(x, y)=f(x, y)$. Define $\bar{g}_{t}: B^{p} \times T V \rightarrow B^{p} \times T Q$ by

$$
\begin{aligned}
\bar{g}_{t}(x, y, z) & =g\left(x, h_{2 t}(y), z\right) & & \text { if }|x| \geqq \frac{1}{2} \text { and } t+|x| \leqq 1 \text { or } \\
& =\hat{g}_{(t+|x|-1) /|x|}\left(x, h_{2(1-|x|)}(y), z\right) & & \text { if }|x| \geqq \frac{1}{2} \text { and } t+|x| \geqq 1, \\
& =\hat{g}_{2 t-1}\left(x, h_{1}(y), z\right) & & \text { if }|x| \leqq \frac{1}{2} \text { and } t \geqq \frac{1}{2} .
\end{aligned}
$$

Then $\bar{g}_{0}=g$ and $\bar{g}_{1}=d \bar{f}$. This completes Case I.

Case II. If $\mathrm{Cl}(M-N)$ is not compact, express it as the union of countably many compact sets $\left\{M_{i}\right\}$ such that for all $i, M_{i} \subset$ Int $M_{i+1}$. The first part of Case I 
tells us how to find a mersion $f_{1}: B^{p} \times U_{1} \rightarrow B^{p} \times Q, U_{1} \cup P_{1}$ being a neighbourhood of $N \cup M_{1}$ in $M^{\prime}$ for some finite set $P_{1}$ such that $f_{1}$ extends $f$ and the mersion $\theta$ of a neighbourhood of $N$, and a homotopy $g_{t, 1}: B^{p} \times T U_{1} \rightarrow B^{p} \times T Q$ from $g$ to $d f_{1}$. Now suppose that $f_{i-1}: B^{p} \times U_{i-1} \rightarrow B^{p} \times Q$ and $g_{t, i-1}: B^{p} \times T U_{i-1} \rightarrow B^{p} \times T Q$ have been defined, $U_{i-1} \cup P_{i-1}$ being a neighbourhood of $N \cup M_{i-1}$ in $M^{\prime}$ for some finite set $P_{i-1}$. Then the first part of Case I enables us to extend $f_{i-1}$ and $g_{t, i-1}$, restricted to some smaller set if necessary, to $f_{i}$ and $g_{t, i}$. Continuing thus, we obtain a mersion $f_{\infty}: B^{p} \times U_{\infty} \rightarrow B^{p} \times Q$ and a homotopy $g_{t, \infty}: B^{p} \times T U_{\infty}$ $\rightarrow B^{p} \times T Q$ satisfying the required conditions, where $U_{\infty} \cup P_{\infty}$ is a neighbourhood of $\bigcup_{i=1}^{\infty} M_{i} \cup N$, i.e. of $M$, in $M^{\prime} ; P_{\infty}$ being some countable set. Thus it remains to extend $f_{\infty}$ and $g_{t, \infty}$ over the set $P_{\infty}$. We proceed as in the second part of Case I. Suppose $x \in P_{\infty}$. If $x$ is in a component of $\mathrm{Cl}(M-N)-N$ whose closure in $M$ is compact, then join $x$ to $M^{\prime}-M$ by an arc $\pi_{x}:[0,1) \rightarrow M^{\prime}-N$. If not, then the following lemma allows us to find a proper arc $\pi_{x}:[0,1) \rightarrow M-N$ so that $\pi_{x}(0)=x$ and $\left\{\mathrm{Cl} \pi_{x}[0,1)\right\} \cap N=\varnothing$. The arcs $\pi_{x}$ may be taken to be disjoint, so that they have disjoint neighbourhoods. Now proceed exactly as in Case I, constructing an isotopy which pushes $U_{\infty} \cup P_{\infty}$ along the arcs $\pi_{x}$ into $U_{\infty}$.

Lemma 20. Suppose $M^{m}$ is a manifold and $N$ is a closed subset of $M$ with $M-N$ a connected set having noncompact closure. Then $\exists$ a proper arc $\pi:[0,1) \rightarrow M$ so that $\{\mathrm{Cl} \pi[0,1)\} \cap N=\varnothing$.

Proof. Choose an open neighbourhood $U$ of $N$ in $M$ so that $M-U$ is connected and $\mathrm{Cl}(M-U)$ is noncompact. To ensure that $M-U$ is connected, we may join the components of $M-U$ by arcs in $M-N$ if necessary and then delete the union of such arcs from $U$. Let $V$ be another such neighbourhood whose closure lies in $U$. Using separability, cover $M-\mathrm{Cl} U$ by countably many open discs. ("Open disc" here means a homeomorph of Int $B^{m}$, the "centre" being some distinguished point of the disc.) Add to this collection countably many discs whose centres lie on $(\mathrm{Cl} U)-U$ and which lie in $M-\mathrm{Cl} V$. By paracompactness, alter the cover if necessary so that no compact subset of $M-V$ meets more than finitely many of of the discs. If the closure in $M$ of any of these discs is noncompact, then a radial arc in such a disc will satisfy the requirements. Thus we may assume that each disc has compact closure in $M$.

Choose a disc $C_{1}$ from the collection and define $\left\{C_{i}\right\}$ inductively as follows. Given $C_{i-1}$, let $C_{i}$ denote the union of those discs in the collection which have not yet been chosen and which meet $C_{i-1}$. Run arcs from the centre of $C_{1}$ to the centres of each of the discs comprising $C_{2}$ such that each of the arcs lies entirely inside the two discs whose centres it joins. Continue this process inductively as follows. Suppose arcs have been constructed to the centres of the discs comprising $C_{i-1}$. Then for each pair of discs, one in $C_{i-1}$ and the other in $C_{i}$, which have a nonempty intersection, construct arcs between their centres, the arcs lying inside the union of the discs. Denote by $A$ the collection of such arcs. 
By connectivity of $M-U$, each disc appears as part of $C_{i}$ for some $i$. Indeed, such a disc can be connected to $C_{1}$ by an arc between their centres. But this arc is compact, so it meets only finitely many of the discs, say $n$ of them. Then certainly the disc is in $C_{i}$ for some $i \leqq n$.

By noncompactness of $M-U$ and compactness of the closures of each of the discs, we have that for all $n, C_{n} \neq \varnothing$. Thus for all $n, \exists$ an arc made up of $n$ segments, i.e. extending into $C_{n}$. Define the arc $\pi$ inductively as follows. $\pi(0)=$ centre of $C_{1}$. Suppose $\pi \mid[0,1-1 / n]$ has been defined for some $n \geqq 1$, with $\pi(1-1 / n)$ the centre of some disc in $C_{n}$, so that for all $i>n, \exists$ an arc in $A$ passing through $\pi(1-1 / n)$ and continuing to $C_{i}$. Now $C_{n+1}$ will have only finitely many discs meeting the disc centred at $\pi(1-1 / n)$ since the closure of this disc is compact and any compact set meets only finitely many of the discs. Thus at least one of these discs will contain arcs of $A$ which proceed arbitrarily far, i.e. to $C_{i}$ for arbitrary $i>n$. Then define $\pi(1-1 /(n+1))$ to be the centre of such a disc in $C_{n+1}$, and let $\pi \mid[1-1 / n, 1-1 /(n+1)]$ be that arc of $A$ joining the two points.

\section{BIBLIOGRAPHY}

1. M. Brown, Locally flat imbeddings of topological manifolds, Ann. of Math. (2) 75 (1962), 331-341. MR 24 \#A3637.

2. R. Edwards and R. Kirby, Deformations of spaces of imbeddings, (to appear).

3. A. Haefliger and V. Poenaru, La classification des immersions combinatoires, Inst. Hautes Études Sci. Publ. Math. No. 23 (1964), 75-91. MR 30 \#2515.

4. M. Hirsch, Immersions of manifolds, Trans. Amer. Math. Soc. 93 (1959), 242-276. MR 22 \#9980.

5. R. Lashof, Lees' immersion theorem and the triangulation of manifolds, Bull. Amer. Math. Soc. 75 (1969), 535-538.

6. J. A. Lees, Immersions and surgeries on topological manifolds, Bull. Amer. Math. Soc. 75 (1969), 529-534.

7. J. P. May, Simplicial objects in algebraic topology, Van Nostrand Math. Studies, no. 11, Van Nostrand, Princeton, N. J., 1967. MR 36 \#5942.

8. A. Phillips, Submersions of open manifolds, Topology 6 (1967), 171-206. MR 34 \#8420.

9. C. Rourke and B. Sanderson, On the homotopy theory of $\Delta$-sets, Ann. of Math. (to appear).

10. S. Smale, The classification of immersions of spheres in Euclidean spaces, Ann. of Math. (2) 69 (1959), 327-344. MR 21 \#3862.

11. R. Kirby and L. Siebenmann, On the triangulation of manifolds and the Hauptvermutung, Bull. Amer. Math. Soc. 75 (1969), 742-749.

The University OF AUCKLAND, Auckland, New Zealand 